\title{
Solving for Mistuned Forced Response by Symmetry
}

\author{
B. Shapiro* \\ California Institute of Technology, Pasadena, California 91125
}

\begin{abstract}
The introduction of mistuning in jet-engine bladed disks can lead to large changes in stability and forced response. Even small random mistuning (within the bounds of manufacturing tolerance) can lead to unacceptable response and high-cycle fatigue. Meanwhile, intentional mistuning may improve stability and forced response under manufacturing uncertainty. This paper presents a general framework for predicting forced response as a function of mistuning. Because the forced response problem is an almostsingular linear problem, its solution is highly nonlinear in the mistuning parameters. Our methods exploit symmetry arguments and eigenstructure perturbation to provide a method valid for any model. It is shown that, by perturbing eigenvectors in the numerator and the inverse of eigenvalues in the denominator (exploiting symmetry in both computations), we can accurately approximate the forced response as a function of mistuning. Results are demonstrated for a simple lightly damped model, and the consequent sharp nonlinear behavior is captured almost perfectly. We also show that intentional mistuning may guarantee improved stability and forced response under fixed manufacturing tolerances. Thus, intentional mistuning should be considered as a practical means of increasing safety and enhancing engine performance.
\end{abstract}

\section{Nomenclature}

$A=A(z)=[i \omega I-M(z)]$, inverse matrix of Eq. (13)

$a=a_{j, d}$, linear eigenvalue perturbation coefficient of Eq. (52)

$\boldsymbol{B}_{l} \quad=$ lth forcing vector, Eq. (11)

$b, c_{i}=b_{j, d}, c_{i}^{j, d}$, quadratic eigenvalue perturbation coefficients of Eq. (52)

$f \quad=\left(f_{1}, f_{2}, \ldots, f_{r}\right)$ cascade model, Eq. (1), $f_{j}$ dynamics of $j$ th blade

$\bar{f} \quad=\bar{f}(x, z)$ unforced system in Eq. (9)

$f^{\prime} \quad=f^{\prime}(x, z, t)$ forced dynamics, Eq. (9)

$L \quad=l \bmod r$, excited modes in tuned response, Eq. (44)

$l \quad=$ spatial mode index in fixed frame, Eq. (11)

$M \quad=M(z)$ linearization of $\bar{f}$ about $x^{*}$, Eq. (12)

$m \quad=$ number of states per blade

$n=r m$, total number of states

$p_{j} \quad=e^{2 \pi i j / r}, j$ th phase coefficient, Theorem 3.1

$r \quad=$ number of blades

$S=S_{j, d}$, quadratic eigenvalue perturbation matrix

$T, T^{-1}=T(z), T^{-1}(z)$ matrix of right, left eigenvectors of $M(z)$, Eq. (34)

$t \quad=$ time

$\boldsymbol{U}_{j}^{d} \quad=\boldsymbol{U}_{j}^{d}(z) \in \mathbb{C}^{n}$ left (row) eigenvector of $M(z)$, in indexing of Theorem 3.1

$\boldsymbol{u}_{j}^{d} \quad=\boldsymbol{u}_{j}^{d}(z) \in \mathbb{C}^{m}$, first $m$ elements or block of $\boldsymbol{U}_{j}^{d}(z)$, Eq. (40)

$\boldsymbol{V}_{j}^{d} \quad=\boldsymbol{V}_{j}^{d}(z) \in \mathbb{C}^{n}$ right (column) eigenvector of $M(z)$, in indexing of Theorem 3.1

$v_{j}^{d} \quad=v_{j}^{d}(z) \in \mathbb{C}^{m}$, first $m$ elements or block of $V_{j}^{d}(z)$, Eq. (40)

$\boldsymbol{X}=\boldsymbol{X}(\boldsymbol{z})$ forced response in frequency domain (13), $\boldsymbol{X}_{j}^{q}$ for $j$ th blade, $q$ th mode

$\boldsymbol{x}=\left(\boldsymbol{x}_{1}, \boldsymbol{x}_{2}, \ldots, \boldsymbol{x}_{r}\right)$ system state, $\boldsymbol{x}_{j} \in \mathbb{R}^{m}$ states for $j$ th blade

$\boldsymbol{x}^{*}=\boldsymbol{x}^{*}(\boldsymbol{z})$ equilibrium point of unforced system $\overline{\boldsymbol{f}}$, Eq. (9)

Received March 18, 1998; revision received July 27, 1998; accepted for publication July 27, 1998. Copyright (C) 1998 by the American Institute of Aeronautics and Astronautics, Inc. All rights reserved.

${ }^{*}$ Graduate Student, Control and Dynamical Systems. E-mail: bshapiro@indra.caltech.edu.

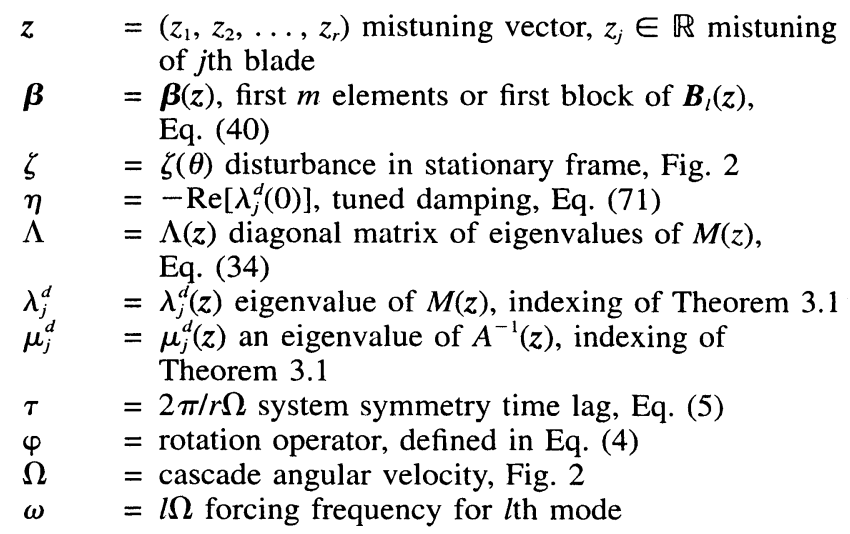

\section{Introduction}

$\mathbf{H}$ IGH-cycle fatigue in titanium engine parts has been identified as the predominant cause of several hundred incidents over the past three decades. ${ }^{1}$ These incidents have ranged from class A mishaps down to maintenance actions. [Class A mishaps involve either 1) property damage in excess of one million dollars, 2) U.S. Air Force aircraft damaged beyond economical repair, or 3) human fatality or a permanent and total disability.] In fact, high-cycle fatigue has been explicitly identified as the dominant unexpected engine failure mode from 1989 to $1994 .^{2}$ Furthermore, fatigue failures are typically dormant: they are identified only after failure has occurred, even though the root cause may have been present for some time. Such data forces an obvious question: what causes these surprise high-cycle fatigue failures?

One potential explanation is blade asymmetry, caused by manufacturing tolerances or wear and tear, which can lead to large asymmetric forced response with resulting high-cycle fatigue. Ideally, fans or compressors are tuned (all blades are identical). In reality, manufacturing along with engine wear creates mistuning (blades are different from one another). It is well known that even a small amount of mistuning can induce a large asymmetric forced response known as mode localization. ${ }^{3,4}$ Only certain mistuning patterns lead to an unacceptable response and, because the majority of engines remain operational, the probability of these patterns occurring must be small. Still, the same probability may be unacceptably large 
by safety standards (we show such an example in Sec. VII). Fatigue because of mistuning is a natural suspect because it displays all of the required symptoms: failure because of an unacceptable mistuning pattern is unexpected (of low probability and probably not seen or predicted by any experiment or analysis), and it is dormant (root cause present for considerable time before failure).

Although random mistuning can lead to failure, intentional mistuning can provide benefits. For one, it can push back flutter margins to allow increased performance. ${ }^{5-9}$ Previous authors have noticed that such an increase in stability can be accompanied by mode localization. As a result, intentional mistuning has been viewed as a tradeoff between a benefit in stability vs a detriment in forced response. It is true that the introduction of intentional mistuning typically creates a larger forced response; however, this misses the point. If intentional mistuning is chosen intelligently, it may lead to robust behavior. In Sec. VII we show a case where the tuned system is not robust: small manufacturing tolerances can lead to disastrous forced response and even instability. Upon the introduction of intentional mistuning, the system becomes robust: now acceptable forced response and stability are guaranteed under the same manufacturing tolerances. Clearly, the latter situation is preferable to the former. Consequently, intentional mistuning (if done properly) might answer the challenge posed by the U.S. Air Force Scientific Advisory Board (AFSAB) ${ }^{1}$ to prevent fatigue failure through better management of vibratory stresses. It is the AFSAB opinion that this task cannot be accomplished through improving resistance of materials to vibratory stresses (except perhaps through composites) or by improved nondestructive evaluations. Hence, other methods must be investigated and we feel that intentional mistuning is one of the more practical alternatives, it makes use of existing technology and does not require new materials or manufacturing techniques.

To this end, we must understand the effect of mistuning on stability and forced response. It is impossible to do so experimentally, as even with just two different blade types the number of combinations grows as $2^{r}$, where $r$ is the number of blades. Consequently, our approach must be analytic. Moreover, it must not only predict behavior for a given mistuning, but it should reveal underlying design tradeoffs to facilitate improved engine design. Figure 1 shows our analysis goal. At the top of the figure we find a first-cut approach: take an engine model $\dot{\boldsymbol{x}}=\boldsymbol{f}(\boldsymbol{x}, z, t)$ and plug in mistuning $z$ to find response $\boldsymbol{X}$. Such an approach is not useful for two reasons: 1) it is computationally intractable (to map out the mistuning space we have to run the possibly complex model $f$ on the order of $2^{r}$ times) and 2) it provides no insight. By comparison, the approach at the bottom of Fig. 1 takes model $\dot{x}=f(x, z$,

Low analysis, high complexity: almost useless.

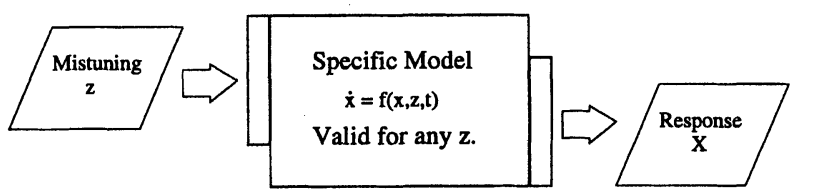

\section{Analysis}

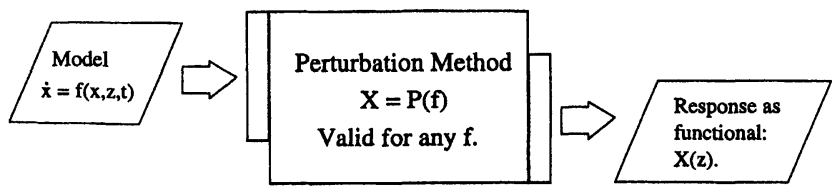

High analysis, low complexity: useful for design.

Fig. 1 Developing a method valid for any model. $t)$ as its input and generates the functional $\boldsymbol{X}(\boldsymbol{z})$ as output. Now we have an (ideally simple) expression for response as a function of mistuning $z$. From expression $X(z)$ we can learn sensitivities and design tradeoffs. Instead of iterating on mistuning, we can iterate on models. Computation complexity has been vanquished. Because the method is model independent, we can gain invaluable understanding on how sensitivity to mistuning varies with parameters and between models. Our paper presents the analysis arrow and achieves the analysis goal at the bottom of Fig. 1, providing a simple accurate functional $X(z)$ that reveals underlying design tradeoffs.

By comparison, previous work ${ }^{7,10}$ varies between the top and middle of Fig. 1. It is not difficult to justify this statement: there are a number of problems considered untractable in the literature that are trivial in our framework. For example, the combinatoric optimization problem of arranging mistuned blades to maximize stability is claimed untractable in the literature, ${ }^{11}$ but is solved closed form in Ref. 9 (albeit in a practical special case). Robustness guarantees under manufacturing tolerances cannot be achieved using current methods in the literature, but are given here in Sec. VII. In Ref. 12, Monte Carlo simulations are performed on a specific model to check the probability of unacceptable forced response (precisely the top approach of Fig. 1). This approach is justified by the claim that all analytic methods (such as in Ref. 13) fail when the system is lightly damped. This is not so. At worst, Monte Carlo simulation can be performed orders of magnitude more efficiently on our functional relation $X(z)$ of Eqs. (43), (66), and (68). At best, these equations will be amenable to analytic estimates of probabilities; certainly we can guarantee a zero probability in some cases by the bounds of Sec. VII. However, our most significant contribution is one of understanding.

We know the symmetric or tuned point is degenerate; it satisfies the rule that all blades are the same. This constrains the eigenvectors or mode shapes to have a constant inter-bladephase-angle. Hence, forcing excites only some of the modes with resulting acceptable behavior. However, even a small amount of mistuning will break the constant inter-blade-angle eigenvector constraint. In that case, the remaining lightly damped modes are exicted, creating a disastrous forced response. Such an understanding reveals the underlying tradeoffs between stability, forced response, and robustness, including how these depend on damping, mode placement, and manufacturing tolerances. These results rest on two mathematical techniques: 1) symmetry arguments and 2) eigenstructure perturbation.

\section{System Symmetry}

Mistuning is fundamentally concerned with symmetry and symmetry breaking. As a result, the symmetry arguments presented in this section are the natural tools of analysis. These arguments formalize the following obvious statement: "Given a tuned system, mistuning blade one and observing blade two is the same as mistuning blade three and observing blade four.' They hold true for every aspect of the problem, whether it is initial model formulation or final performance optimization. In our analysis, symmetry is applied to any dynamic discrete-blade cascade model of the form $\dot{x}=f(x)$. Symmetry arguments also apply to imposed sinusoidal motion models that correspond to the special case $\boldsymbol{x}$ restricted to $\boldsymbol{x}(t)=\overline{\boldsymbol{x}}+$ $\boldsymbol{x}^{\prime} e^{i \omega t}$. In fact, conclusions achieved by symmetry considerations apply even when $\boldsymbol{f}(\boldsymbol{x})$ cannot be written explicitly, as is the case in computational fluid dynamic (CFD) models.

Suppose a fan or compressor has $r$ blades. Start with any cascade flutter model of the form

$$
\dot{\boldsymbol{x}}=\boldsymbol{f}(\boldsymbol{x}, z, t)
$$

where $\boldsymbol{x}=\left(\boldsymbol{x}_{1}, \boldsymbol{x}_{2}, \ldots, \boldsymbol{x}_{r}\right) \equiv \mathbb{R}^{r m}$ is the state vector with $\boldsymbol{x}_{i} \in$ $\mathbb{R}^{m}$ corresponding to aerodynamic and structural states for the $i$ th blade. Mistuning is represented by the vector $z \in \mathbb{R}^{r}$. An 
element $z_{i} \in \mathbb{R}$ denotes mistuning for the $i$ th blade. For example, if we mistune the stiffness of individual blades, then define the $i$ th blade stiffness $k_{i}=k_{0}\left(1+z_{i}\right)$, where $k_{0}$ is the nominal or tuned stiffness. As in this example, $z=0$ will always denote the tuned case. Finally, $t$ represents time because we allow time-dependent forcing.

Consider the dynamics of the first blade:

$$
\dot{\boldsymbol{x}}_{1}=\boldsymbol{f}_{1}\left(\boldsymbol{x}_{1}, \boldsymbol{x}_{2}, \ldots, \boldsymbol{x}_{r}, z_{1}, z_{2}, \ldots, z_{r}, t\right)
$$

which correspond to the first block row (the first $m$ rows) of the vector field $\boldsymbol{f}$ in Eq. (1). Clearly, the dynamics of the first blade $\left(\dot{\boldsymbol{x}}_{1}\right)$ depend on the state of the third blade $\left(\boldsymbol{x}_{3}\right)$, the same way the dynamics of the second blade $\left(\dot{x}_{2}\right)$ depend on the state of the fourth blade $\left(\boldsymbol{x}_{4}\right)$. The same holds for the mistuning vector $z$, the dynamics of the first blade $\left(\dot{\boldsymbol{x}}_{1}\right)$ depend on the mistuning of the seventh blade $\left(z_{7}\right)$, the same way the dynamics of the third blade $\left(\dot{\boldsymbol{x}}_{3}\right)$ depend on the mistuning of the ninth blade $\left(z_{9}\right)$. Applying this chain of logic to all blades, we must have

$$
\begin{aligned}
\dot{\boldsymbol{x}}_{1}= & \boldsymbol{f}_{1}(\boldsymbol{x}, z, t) \\
= & \boldsymbol{f}_{1}\left(\boldsymbol{x}_{1}, \boldsymbol{x}_{2}, \boldsymbol{x}_{3}, \ldots, \boldsymbol{x}_{r-1}, \boldsymbol{x}_{r}, z_{1}, z_{2}, z_{3}, \ldots, z_{r-1}, z_{r}, t\right) \\
\dot{\boldsymbol{x}}_{2}= & \boldsymbol{f}_{2}(\boldsymbol{x}, z, t) \\
= & \boldsymbol{f}_{1}\left[\boldsymbol{x}_{2}, \boldsymbol{x}_{3}, \ldots, \boldsymbol{x}_{r-1}, \boldsymbol{x}_{r}, \boldsymbol{x}_{1}, z_{2}, z_{3}, \ldots, z_{r-1}, z_{r}, z_{1}, t\right. \\
& +(2 \pi / r \Omega)] \\
\dot{\boldsymbol{x}}_{3}= & \boldsymbol{f}_{3}(\boldsymbol{x}, z, t) \\
= & \boldsymbol{f}_{1}\left[\boldsymbol{x}_{3}, \boldsymbol{x}_{4}, \ldots, \boldsymbol{x}_{r}, \boldsymbol{x}_{1}, \boldsymbol{x}_{2}, z_{3}, z_{4}, \ldots, z_{r}, z_{1}, z_{2}, t\right. \\
& +(4 \pi / r \Omega)] \\
\vdots & \\
\dot{x}_{r}= & f_{r}(\boldsymbol{x}, z, t) \\
= & f_{1}\left\{\boldsymbol{x}_{r}, \boldsymbol{x}_{1}, \ldots, \boldsymbol{x}_{r-2}, \boldsymbol{x}_{r-1}, z_{r}, z_{1}, \ldots, z_{r-2}, z_{r-1}, t\right. \\
& +[2 \pi(r-1) / r \Omega]\}
\end{aligned}
$$

The time shift of $2 \pi / r \Omega$ in expression (3) follows from assuming a stationary disturbance, as would be generated by stator/ inlet guide vane wakes or by an inlet distortion. For a cascade of undeformed blades rotating at angular velocity $\Omega$ past such a stationary disturbance (as in Fig. 2), the disturbance at blade two will precede the same disturbance at blade one by $2 \pi / r \Omega$. Now consider the case where blades are deforming because of their states $\boldsymbol{x}$ and possibly their mistuning $z$ (for example, mistuning stiffness will change the nominal deformation of blades under aerodynamic loading). If at time $t$, blade one is deformed from its nominal angular position $\theta_{1}$ by some amount $\psi$ because of its state $\left(x_{1}=\alpha\right)$ and its mixtuning $\left(z_{1}=\beta\right)$, then it occupies the position $\theta_{1}+\psi(\alpha, \beta)$ and has some shape deformation $\zeta(\alpha, \beta)$. It follows that if blade two has the same state $\left(\boldsymbol{x}_{2}=\boldsymbol{\alpha}\right)$ and mistuning $\left(z_{2}=\beta\right)$ at time $t+2 \pi / r \Omega$, then it must deform an identical amount and will also occupy the position $\theta_{1}+\psi(\boldsymbol{\alpha}, \beta)$ with the same shape $\zeta(\boldsymbol{\alpha}, \beta)$. At these two identical conditions (same position and shape), blades one and two will experience the same dynamics because of forcing. This is precisely the statement in Eq. (3), $\dot{x}_{1}$ depends on $x_{1}, z_{1}$, and $t$ the same way $\dot{x}_{2}$ depends on $x_{2}, z_{2}$, and $t+2 \pi / r \Omega$.

To ease notation we define the rotation operator $\varphi$, which rotates vectors of size $m$ or $r m$ to the left. The scope of the argument (size $r$ or $r m$ ) is clear from context:

$$
\begin{array}{r}
\varphi^{j}\left(z_{1}, z_{2}, \ldots, z_{r}\right)=\left(z_{1+j}, z_{2+j}, \ldots, z_{r}, z_{1}, \ldots, z_{j}\right) \\
z_{i} \in \mathbb{R} \text { or } \mathbb{C} \\
\varphi^{j}\left(\boldsymbol{x}_{1}, \boldsymbol{x}_{2}, \ldots, \boldsymbol{x}_{r}\right)=\left(\boldsymbol{x}_{1+j}, \boldsymbol{x}_{2+j}, \ldots, \boldsymbol{x}_{r}, \boldsymbol{x}_{1}, \ldots, \boldsymbol{x}_{j}\right) \\
\boldsymbol{x}_{i} \in \mathbb{R}^{m} \text { or } \mathbb{C}^{m}
\end{array}
$$

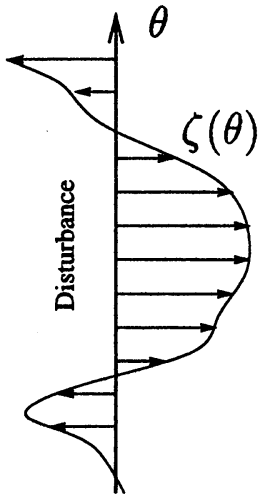

Stationary Frame

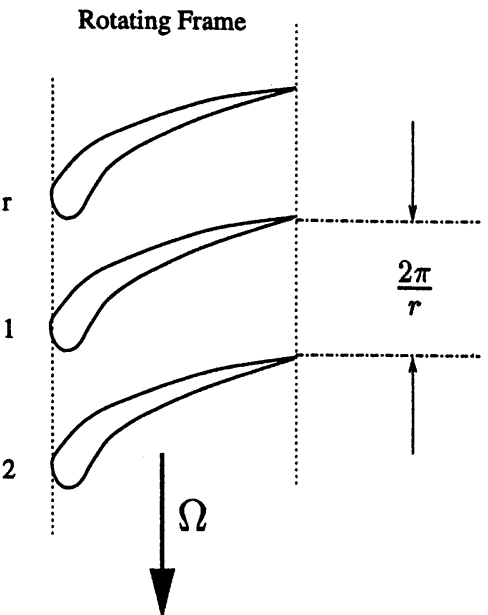

Fig. 2 Cascade disturbance.

For convenience, $\varphi^{1}$ is written simply as $\varphi$, and $\varphi^{-1}$ is the inverse rotation to the right. Using the rotation operator and defining $\tau=2 \pi / r \Omega$, condition (3) on model (1) can be rewritten succinctly as

$$
\varphi f(x, z, t)=f(\varphi x, \varphi z, t+\tau)
$$

Applying Eq. (5) a total of $r$ times, we note $f(x, z, t)=f(x$, $z, t+2 \pi / \Omega)$, which is of course the periodicity associated with a complete rotation. As shown in Fig. 2, we have assumed that the disturbance $\zeta(\theta)$ is constant in the stationary frame.

Expression (5) is a system symmetry and is the basic building block for all of the analysis that follows. Let $\xi(\hat{x}, s, z, t)$ denote trajectories or solutions to Eq. (1), with initial condition $\hat{\boldsymbol{x}}$ at time $s$, where the mistuning has been fixed at $\boldsymbol{z}$. For this initial condition $\hat{\boldsymbol{x}}$ at time $t=s$ [so $\boldsymbol{x}(s)=\boldsymbol{\xi}(\hat{\boldsymbol{x}}, s, z, s)=\hat{\boldsymbol{x}}$ ], the state of the system at any other time $t$ is given by $x(t)=$ $\boldsymbol{\xi}(\hat{\boldsymbol{x}}, s, \boldsymbol{z}, t)$. It follows from Eq. (5) that solutions $\boldsymbol{\xi}$ must satisfy

$$
\varphi \boldsymbol{\xi}(\hat{\boldsymbol{x}}, s, z, t)=\boldsymbol{\xi}(\varphi \hat{\boldsymbol{x}}, s+\tau, \varphi z, t+\tau)
$$

For a proof of this, see Theorem A.1. Equation (6) says that, if we know the solution for $\hat{\boldsymbol{x}}, s$, and $\boldsymbol{z}$, then we have also found the trajectory for initial condition $\varphi \hat{x}$ at time $s+\tau$ with mistuning $\varphi z$.

In the special case of a tuned cascade $\left(\varphi z=z\right.$ or $z_{1}=z_{2}=$ $\cdots=z_{r}$ ) and no forcing [no time dependence in Eq. (1)], we can drop the now redundant $z$ and $t$ notation to reduce Eq. (5) to

$$
f(x)=\varphi^{-1} f(\varphi x)
$$

If we further pick tuned initial conditions $\varphi \hat{x}=\hat{\boldsymbol{x}}$, then Eq. (6) becomes ( $s$ is dropped because all starting times are equivalent)

$$
\varphi \boldsymbol{\xi}(\hat{\boldsymbol{x}}, t)=\boldsymbol{\xi}(\hat{\boldsymbol{x}}, t)
$$

which implies $\xi_{1}(\hat{\boldsymbol{x}}, t)=\xi_{2}(\hat{\boldsymbol{x}}, t)=\cdots=\boldsymbol{\xi}_{r}(\hat{\boldsymbol{x}}, t)$, and the solution remains tuned for all time.

In the remainder of this work we study the leading-order linear problem (see Sec. III.B), and so exploit a special case of Eqs. (5) and (6). Nevertheless, it is instructive to note the scope of symmetry arguments that provide results for the full nonlinear system [Eq. (1)]. Interested readers are referred to 
Golubitsky et al. ${ }^{14}$ for a deeper analysis of symmetry tools and their application to dynamical systems.

\section{Linearization and Symmetries}

\section{A. Assumptions}

To show the assumptions made, break up the full forced nonlinear system [Eq. (1)] into a forced and unforced part:

$$
\dot{x}=f(x, z, t)=\bar{f}(x, z)+f^{\prime}(x, z, t)
$$

here $\bar{f}$ is the dynamics of Eq. (1) if there is no disturbance, and $\boldsymbol{f}^{\prime}$ is defined as $\boldsymbol{f}-\overline{\boldsymbol{f}}$. Now consider an equilibrium point $x^{*}(z)$ of the unforced system, $\bar{f}\left[x^{*}(z), z\right]=0$. (It is crucial to note that $x^{*}$ can vary with mistuning; for instance, a change in stiffness will cause different nominal deformations in blades because of aerodynamic loading.)

It seems valid to assume that the equilibrium point of interest $\boldsymbol{x}^{*}$ for the tuned unforced system $\overline{\boldsymbol{f}}(\boldsymbol{x}, 0)$ is symmetric, $x^{*}(0)=\varphi x^{*}(0)$, because, if this assumption does not hold, then we must have a whole family of equilibria: $x_{1}^{*}(0)=x^{*}(0)$, $\boldsymbol{x}_{2}^{*}(0)=\varphi \boldsymbol{x}^{*}(0), \boldsymbol{x}_{3}^{*}(0)=\varphi^{2} \boldsymbol{x}^{*}(0), \cdots, \boldsymbol{x}_{r}^{*}(0)=\varphi^{r-1} \boldsymbol{x}^{*}(0)$. See Lemma A.1, which is an occurrence not seen in jet engines. Linearization of Eq. (9) about $\boldsymbol{x}^{*}(z)$ yields the time-varying linear dynamics:

$$
\dot{x}=\{\underbrace{\frac{\partial \bar{f}}{\partial x}\left[x^{*}(z), z\right]}_{\text {time invariant }}+\underbrace{\frac{\partial f^{\prime}}{\partial x}\left[x^{*}(z), z, t\right]}_{\text {time varying }}\}\left[x-x^{*}(z)\right]+f^{\prime}\left[x^{*}(z), z, t\right]
$$

As an example, in simple aerodynamic models, the force on blade $i$ is proportional to the distance between adjacent blades times the angle of attack: $\sin (\alpha)\left[x_{i+1}-x_{i-1}\right]$. An inlet distortion changes the incoming velocity in the fixed frame, and so, spinning blades see a resulting variation in angle of attack as a function of time, $\alpha(t)=\alpha+\Delta(t)$. This results in the linear time-varying perturbation term $\{\sin [\alpha+\Delta(t)]-\sin (\alpha)\}\left(x_{i+1}\right.$ $\left.-x_{i-1}\right)$. Even though such terms are physically of second order [the first-order effect is clearly $\sin (\alpha)\left(x_{i+1}-x_{i-1}\right)$ ], they are perturbing a lightly damped matrix $(\partial \bar{f} / \partial x)$ (turbomachines have lightly damped modes), and so, may cause large changes. Clearly, one must solve the time-invariant problem before attempting the more difficult time-varying case, and so, we neglect the $\left[\partial \overline{\boldsymbol{f}}^{\prime} / \partial \boldsymbol{x}\right]$ term in the remainder. However, future work should examine the effect of the time-varying $\left[\partial \bar{f}^{\prime}(\ldots, t) / \partial x\right]$ term and check whether it can be ignored.

\section{B. Linear Problem}

To leading order, forced response is a linear problem. Two analytic methods are required to solve the problem: the first is a symmetry arguments toolbox as addressed in Sec. II and specialized to linear systems later in this section; the second is a set of linear algebra tools dealing with perturbations of eigenvalues and eigenvectors (Sec. V). In this section we discuss the first method.

Neglecting higher-order terms (including the time-varying linear term $\left[\partial \boldsymbol{f}^{\prime}(\ldots, t) / \partial \boldsymbol{x}\right]$, as described in Sec. III.A) we get the standard linear problem:

$$
\dot{\boldsymbol{x}}=M(z) \boldsymbol{x}+\boldsymbol{B}_{l}(z) e^{i l \Omega t}
$$

Here

$$
M(z) \triangleq\left\{\frac{\partial \bar{f}}{\partial x}[x *(z), z]\right\}
$$

and the deviation from equilibrium $x-x *(z)$ has been relabeled as $\boldsymbol{x}$. Coefficient $\boldsymbol{B}_{l}$ corresponds to the $l$ th spatial mode of the disturbance in the fixed reference frame: $\zeta(\theta)=\sin (l \theta)$ in Fig. 2 with resulting forcing frequency $\omega=l \Omega$.

For $M(z)$ stable (see Ref. 9), the transient response decays to zero and the steady-state forced response may be written in frequency domain as

$$
\boldsymbol{X}(z)=[i \omega I-M(z)]^{-1} \boldsymbol{B}_{l}(z)
$$

Here, steady-state forced response in the time domain is given by $\overline{\boldsymbol{x}}(z, t)=\boldsymbol{X}(\boldsymbol{z}) e^{i \omega t}$. Because analysis is conducted at a fixed spatial mode $l, X$ dependence on $l$ and $\omega$ is understood but dropped in the notation.

Up to our linear time-invariant formulation, expression (13) provides the exact forced response for any specific mistuning $z$. Yet Eq. (13) by itself is not useful for two reasons.

1) It is computationally infeasible to re-evaluate Eq. (13) for each new mistuning pattern; typical cascade models have many states and require long computation times. Consequently, it is impractical to characterize response to mistuning by computing Eq. (13) for a large set of mistuning patterns.

2) Computing Eq. (13) for each new pattern $z$ provides almost no understanding. Sensitivity and parameter dependence is not revealed by exhaustive calculation.

Instead, one should find an approximation of the forced response as a function of mistuning, $\boldsymbol{X}(z) \approx \bar{X}(z)$, where $\bar{X}$ is tractable and provides insight. To do so, we must approximate the inverse of $A(z)=[i \omega I-M(z)]$ in Eq. (13) as a function of $z$. In Sec. IV we find that because $A(z)$ is almost singular, its inverse is a steep nonlinear function of $z$. Thus, approximating this inverse matrix is at once our main aim and the major difficulty.

Toward this aim we now discuss symmetries of Eq. (13). In Lemma A.3, we show how symmetry (5) along with equilibrium symmetry $\varphi x^{*}(z)=x^{*}(\varphi z)$ (see Sec. III.A and Lemma A.2) imply a symmetry on the linearization $M$ :

$$
\varphi M(z)=M(\varphi z) \varphi
$$

This is to be interpreted as follows: for any vector $\boldsymbol{x}$

$$
\varphi[M(z) x]=M(\varphi z)(\varphi x)
$$

and so, $\varphi$ only acts on vectors as defined in Eq. (4). If we now let $z=0$, we find that $M(0)$ must satisfy $M(0)=\varphi^{-1} M(0) \varphi$, and so, it is trivial to check that the tuned linearization $M(0)$ must be block circular. Precisely, $\bar{M}=M(0)$ must have the form

$$
\bar{M}=\left[\begin{array}{cccccc}
\bar{M}_{1} & \bar{M}_{2} & \bar{M}_{3} & \cdots & \bar{M}_{r-1} & \bar{M}_{r} \\
\bar{M}_{r} & \bar{M}_{1} & \bar{M}_{2} & \bar{M}_{3} & \cdots & \bar{M}_{r-1} \\
& \ddots & \ddots & \ddots & \ddots & \\
\bar{M}_{2} & \bar{M}_{3} & \cdots & \bar{M}_{r-1} & \bar{M}_{r} & \bar{M}_{1}
\end{array}\right]
$$

where $\bar{M}_{j} \in \mathbb{R}^{m \times m}$.

The structure in Eq. (16) motivates Theorem 3.1, which is crucial in analysis and computation. Now let $p_{j}=\exp (2 \pi i j / r)$ denote powers of the $r$ th root of unity, where $i=\sqrt{-1}$, and define

$$
\begin{array}{r}
Q_{j}=\bar{M}_{1}+p_{j} \bar{M}_{2}+p_{j}^{2} \bar{M}_{3}+\cdots+p_{j}^{r-1} \bar{M}_{r} \in \mathbb{C}^{m \times m} \\
j \in(1,2, \ldots, r)
\end{array}
$$

Theorem 3.1: For $\bar{M}$ a block circular matrix as in Eq. (16), let $\lambda_{j}^{d}, \boldsymbol{u}_{j}^{d}$, and $\boldsymbol{v}_{j}^{d}$ be the $d$ th eigenvalue, left eigenvector, and right eigenvector, respectively, of $Q_{j}$. Then $\left(\lambda_{j}^{d}\right)$ form eigenvalues of $\bar{M}$ with left and right eigenvectors $\boldsymbol{U}_{j}^{d}=\left(\boldsymbol{u}_{j}^{d}\right.$, $\left.p_{j}^{r-1} \boldsymbol{u}_{j}^{d}, p_{j}^{r-2} \boldsymbol{u}_{j}^{d}, \ldots, p_{j} \boldsymbol{u}_{j}^{d}\right)$ and $\boldsymbol{V}_{j}^{d}=\left(v_{j}^{d}, p_{j} \boldsymbol{v}_{j}^{d}, \ldots, p_{j}^{r-2} \boldsymbol{v}_{j}^{d}\right.$, $\left.p_{j}^{r-1} \boldsymbol{v}_{j}^{d}\right)$. 
For proof of the preceding theorem, please see the Appendix. This theorem also proves the constant inter-blade-phaseangle assumption (corresponding to preceding symmetric eigenvectors with phase angle $p_{j}$ ), common in flutter analysis, which only holds when the system is tuned. Matrix symmetry (14), together with Theorem 3.1 , imply a symmetry on the mistuned right and left eigenvectors of $M(z)$ :

$$
\begin{gathered}
\boldsymbol{V}_{j}^{d}(\boldsymbol{z})=p_{j} \varphi^{-1} \boldsymbol{V}_{j}^{d}(\varphi z) \\
\boldsymbol{U}_{j}^{d}(\boldsymbol{z})=p_{j}^{-1} \varphi^{-1} \boldsymbol{U}_{j}^{d}(\varphi z)
\end{gathered}
$$

with notation of Theorem 3.1 (see Lemma A.6). Recall that right and left eigenvectors, $\boldsymbol{V}$ and $\boldsymbol{U}$, are defined by $\boldsymbol{M V}=\lambda \boldsymbol{V}$ and $\boldsymbol{U}^{T} M=\lambda \boldsymbol{U}^{T}$, respectively. Proofs of earlier statements, Theorem 3.1 and Lemmas A.3 and A.6, may be found in the Appendix.

In fact, every object associated with Eq. (13) inherits a symmetry from the nonlinear symmetry Eq. (5). For instance, eigenvalues $\lambda(z)$ of $M(z)$ have an invariance symmetry, Lemma A.5:

$$
\lambda(z)=\lambda(\varphi z)
$$

Physically, if we change the first blade or the 10th blade (a rotation by nine of $z$ ), our eigenvalues (and, hence, quantities such as minimum damping) will remain the same. Eigenvalue invariance is the fundamental assumption made in our stability paper. ${ }^{9}$

Perhaps the most important symmetry of all is that of the forced response, Lemma A.7:

$$
X(z)=p_{l} \varphi^{-1} X(\varphi z)
$$

Hence, if we know the first blade dynamics $\boldsymbol{X}_{1}(z)$ for arbitrary mistuning $z$ we know the entire response by

$$
\begin{aligned}
\boldsymbol{X}_{2}(z) & =p_{l} \boldsymbol{X}_{1}(\varphi z) \\
\boldsymbol{X}_{3}(z) & =p_{l}^{2} \boldsymbol{X}_{1}\left(\varphi^{2} z\right) \\
& \vdots \\
\boldsymbol{X}_{r}(z) & =p_{l}^{r-1} \boldsymbol{X}_{1}\left(\varphi^{r-1} z\right)
\end{aligned}
$$

This means that instead of having to approximate the entire forced response $\boldsymbol{X}$, we only have to find $\boldsymbol{X}_{1}(z)$ and all other blades $\boldsymbol{X}_{2}, \ldots, \boldsymbol{X}_{r}$ follow from Eq. (21). This is true for all of the symmetries (linearization $M$, eigenvectors $V$ and $U$, eigenvalues $\lambda$, forcing vector $\boldsymbol{B}_{l}$ and forced response $\boldsymbol{X}$ ), they allow us to find the entire object for any mistuning $z$ by computing a single blade or block as a functional form. To conclude, we list all relevant symmetries in Table 1 .

\section{Perturbation Comparisons}

We compare two methods of approximating the inverse ma$\operatorname{trix} A^{-1}(z)=[i \omega I-M(z)]$. Method one is a series expansion whose variations are used in much of the mode localization literature. Method two is based on eigenvalue/vector perturbations and is developed in Sec. V.

\section{A. Series Approximation}

If the spectral radius of matrix $P$ is less than one, $\rho(P)<1$, meaning that all eigenvalues of $P$ lie within the open unit disk, $|\lambda|<1$, then the following series converges:

$$
(I+P)^{-1}=I-P+P^{2}-P^{3}+\cdots
$$

Series (22) may be used to approximate the inverse of matrix $A(z)=[i \omega I-M(z)]$ in Eq. (13). In shorthand, let $A_{0}=A(0)$ and $\Delta A_{z}=A(z)-A(0)$; then

$$
\begin{aligned}
A^{-1}(z) & =\left(A_{0}+\Delta A_{z}\right)^{-1} \\
& =\left[A_{0}\left(I+A_{0}^{-1} \Delta A_{z}\right)\right]^{-1} \\
& =\left(I+A_{0}^{-1} \Delta A_{z}\right)^{-1} A_{0}^{-1} \\
& =\left[I-A_{0}^{-1} \Delta A_{z}+\left(A_{0}^{-1} \Delta A_{z}\right)^{2}-\cdots\right] A_{0}^{-1}
\end{aligned}
$$

which converges if and only if $\rho\left(A_{0}^{-1} \Delta A_{z}\right)<1$. A fundamental problem is that $A_{0}$ is almost singular. Engines have light damping, $\left[\lambda_{j}(0)\right] \approx 0$, and can be forced near resonance, $\omega \approx$ $\operatorname{Re} \operatorname{Im}\left[\lambda_{j}(0)\right]$. Hence, $A(0)$ can have an almost zero eigenvalue: $i \omega-\lambda_{j}(0) \approx 0$. Consequently, $A_{0}^{-1}$ is very large, and so $\rho\left(A_{0}^{-1} \Delta A_{z}\right)<1$ only allows very small matrices $\Delta A_{z}$ and, hence, only a small range of $z$ is permitted. Thus, the series method has a tiny region of validity when the system is lightly damped and forced close to resonance, as is the case in turbomachines.

\section{B. Eigenvalue/Vector Approximation}

The inverse matrix $A^{-1}(z)$ can be written as

$$
A^{-1}(z)=[i \omega I-M(z)]^{-1}=T(z)[i \omega I-\Lambda(z)]^{-1} T^{-1}(z)
$$

where

$$
[i \omega I-\Lambda(z)]^{-1}=\left[\begin{array}{ccc}
\frac{1}{i \omega-\lambda_{1}(z)} & & 0 \\
& \ddots & \\
0 & & \frac{1}{i \omega-\lambda_{n}(z)}
\end{array}\right]
$$

Here $\lambda(z), T(z)$, and $T^{-1}(z)$ are the eigenvalues and right/left eigenvectors of linearization $M(z)$. By approximating eigenvalues $\lambda(z)$, we can easily capture matrix (25), even when $A(z)$ is almost singular: $i \omega-\lambda_{j}(z) \approx 0$.

Table 1 System symmetries ${ }^{a}$

\begin{tabular}{lcccc}
\hline \hline Object & Symbol & Symmetry & Equation & Proof \\
\hline Model & $\boldsymbol{f}$ & $\boldsymbol{f}(\boldsymbol{x}, \boldsymbol{z}, t)=\varphi^{-1} \boldsymbol{f}(\varphi \boldsymbol{x}, \varphi z, t+\tau)$ & $(1)$ & Sec. II \\
Unforced model & $\bar{f}$ & $\bar{f}(\boldsymbol{x}, \boldsymbol{z})=\varphi^{-1} \bar{f}(\varphi \boldsymbol{x}, \varphi z)$ & $(9)$ & Trivial \\
Trajectories & $\boldsymbol{\xi}$ & $\boldsymbol{\xi}(\hat{\boldsymbol{x}}, s, z, t)=\varphi^{-1} \boldsymbol{\xi}(\varphi \hat{\boldsymbol{x}}, s+\tau, \varphi z, t+\tau)$ & $(6)$ & Theorem A.1 \\
Equilibrium & $\boldsymbol{x}^{*}$ & $\boldsymbol{x}(\boldsymbol{z})=\varphi^{-1} \boldsymbol{x}^{*}(\varphi z)$ & $(10)$ & Lemma A.2 \\
Response & $\boldsymbol{X}$ & $\boldsymbol{X}(\boldsymbol{z})=p_{l} \varphi^{-1} \boldsymbol{X}(\varphi z)$ & $(13)$ & Lemma A.7 \\
Forcing vector & $\boldsymbol{B}_{l}$ & $\boldsymbol{B}_{l}(\boldsymbol{z})=p_{l} \varphi^{-1} \boldsymbol{B}_{l}(\varphi z)$ & $(13)$ & Lemma A.4 \\
Linearization & $M$ & $M(z)=\varphi^{-1} M(\varphi z) \varphi$ & $(13)$ & Lemma A.3 \\
Eigenvalues & $\lambda$ & $\lambda(z)=\lambda(\varphi z)$ & $(37)$ & Lemma A.5 \\
Right eigenvectors & $\boldsymbol{V}_{j}^{d}$ & $\boldsymbol{V}_{j}^{d}(\boldsymbol{z})=p_{j} \varphi^{-1} \boldsymbol{V}_{j}^{d}(\varphi z)$ & $(37)$ & Lemma A.6 \\
Left eigenvectors & $\boldsymbol{U}_{j}^{d}$ & $\boldsymbol{U}_{j}^{d}(\boldsymbol{z})=p_{j}^{-1} \varphi^{-1} \boldsymbol{U}_{j}^{d}(\varphi z)$ & $(37)$ & Lemma A.6 \\
\hline \hline
\end{tabular}

${ }^{\mathrm{a}} p_{j}=\exp (2 \pi i j / r)$. 
A fundamental limitation of this method is eigenvalue collision. When eigenvalues are indistinct, $\lambda_{j}(z)=\lambda_{i}(z)$, eigenvectors become discontinuous and the eigenvector perturbations of Sec. V fail. If $M(0)$ has almost indistinct eigenvalues, then small $z$ may cause these eigenvalues to collide on some surface that passes close to the origin, and our perturbation technique (which begins at the origin $z=0$ ) will not be able to see close to or past this surface. A concrete example of this phenomena is demonstrated in Sec. IV.C.

As noticed by Mignolet and $\mathrm{Hu}^{15}$ and others, it is difficult to check the series convergence condition $\rho\left(A_{0}^{-1} \Delta A_{z}\right)<1$ for general matrices $A_{0}$ and mistuning $z$. It is even more difficult to check the distinct condition of the eigenvalue/vector method. However, this does not prevent us from checking these conditions for a simple example. We do so next and note that method limitations revealed by the example generalize to arbitrary linear systems.

\section{Example: Series vs Eigenvalue/Vector Convergence}

Take the simplest possible case: two blades, $r=2$, with one state per blade, $m=1$. Pick the following matrix $A(z)=[i \omega I$ - $M(z)$ ], with symmetry (14):

$$
A(z)=\left[\begin{array}{ll}
a+z_{1} & b+z_{2} \\
b+z_{1} & a+z_{2}
\end{array}\right]
$$

where coefficient $a$ may be complex (it depends on $i \omega$ ), but $z_{1}, z_{2}$, and $b$ are real. Matrix (26) has eigenvalues

$$
\lambda_{1}(z)=a-b, \quad \lambda_{2}(z)=a+b+z_{1}+z_{2}
$$

with right (column) eigenvectors

$$
V_{1}(z)=\left[\begin{array}{c}
b+z_{2} \\
-b-z_{1}
\end{array}\right], \quad V_{2}(z)=\left[\begin{array}{l}
1 \\
1
\end{array}\right]
$$

and left (row) eigenvectors

$$
\boldsymbol{U}_{1}(z)=\frac{(1,-1)}{2 b+z_{1}+z_{2}}, \quad \boldsymbol{U}_{2}(z)=\frac{\left(b+z_{1}, b+z_{2}\right)}{2 b+z_{1}+z_{2}}
$$

The inverse of $A(z)$ is given by

$$
\begin{aligned}
& A^{-1}(z)=1 /\left[(a-b)\left(a+b+z_{1}+z_{2}\right)\right] \\
& \quad \times\left[\begin{array}{cc}
a+z_{2} & -b-z_{2} \\
-b-z_{1} & a+z_{1}
\end{array}\right]
\end{aligned}
$$

We see immediately that $A^{-1}(z)$ has a steep nonlinear dependence on $z$ if $a+b \approx 0$, precisely if $A(0)$ has an eigenvalue close to zero: $\lambda_{2}(0) \approx 0$.

It is trivial to check convergence conditions of the series and eigenvalue/vector methods for this example. Matrix $A_{0}^{-1} \Delta A_{z}$ in Eq. (23) has eigenvalues:

$$
\zeta_{1}=0, \quad \zeta_{2}=\left(z_{1}+z_{2}\right) /(a+b)
$$

Hence the condition for convergence of the series method is given by

$$
\rho\left(A_{0}^{-1} \Delta A_{z}\right)<1 \Leftrightarrow\left|z_{1}+z_{2}\right|<|a+b|
$$

Expression (31) is a conservative guarantee that $\lambda_{2}(z) \neq 0$ in Eq. (27).

On the other hand, our eigenvalue/vector perturbation that begins at the origin, $z=0$, will not be able to see across the $1 /\left(2 b+z_{1}+z_{2}\right)$ singularity in the eigenvectors (29). Hence, the eigenvalue/vector method only converges if

$$
\begin{array}{ll}
z_{1}+z_{2}>-2 b & \text { when } \quad b \geq 0 \\
z_{1}+z_{2}<-2 b & \text { when } \quad b<0
\end{array}
$$

where $b$ is real. This is exactly the point where eigenvalues become indistinct:

$$
\lambda_{2}(z)-\lambda_{1}(z)=2 b+z_{1}+z_{2}=0
$$

If $A(0)$ is almost-singular $\left[\lambda_{2}(0)=a+b \approx 0\right]$, but the eigenvalues are far from being indistinct $\left[\lambda_{1}(0)-\lambda_{2}(0)=2 b\right.$ $\approx 0$ ], then the series approximation has a tiny silver of convergence while the eigenvalue/vector method has a large region of convergence (Fig. 3). [Shading denotes regions where Eqs. (31) or (32) fail and methods do not converge.] This is a case we expect to see in practice. For the contrary case: nearly indistinct eigenvalues $\left[\lambda_{1}(0)-\lambda_{2}(0)=2 b \approx 0\right]$, but far from singularity $\left[\lambda_{1}(0), \lambda_{2}(0) \approx 0\right.$, hence, $\left.a \approx 0\right]$, the series method is good and the eigenvalue/vector method is bad (Fig. 4). For the case where we have indistinct and almost-singular eigenvalues $\left[\lambda_{1}(0) \approx \lambda_{2}(0) \approx 0\right.$ or $a \approx b \approx 0$ ], both methods are poor (Fig. 5). This can also be the case. For example: compressors with many small rigid blades may exhibit closely spaced, lightly damped modes.

It should be noted that variations and enhancements on the series method will still be poor in the almost singular case. Fundamentally, it is not possible to approximate the (multivariable) discontinuous function $1 /\left[i \omega-\lambda_{j}(z)\right]$ by a smooth polynomial expression

$$
1 /\left[i \omega-\lambda_{j}(z)\right] \approx a_{0}+a_{1} \cdot z+z^{T} A_{2} z+\cdots
$$

in a substantial region of $z$ space when $i \omega-\lambda_{j}(0)$ is small. Yet, it is possible to achieve an approximation that holds over

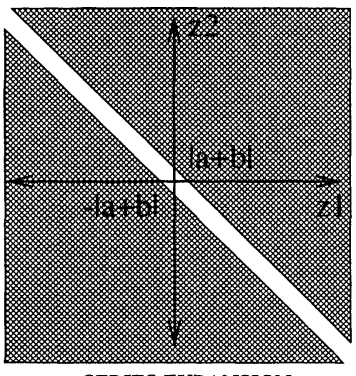

SERIES EXPANSION

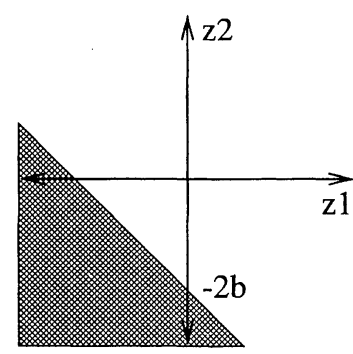

EIGENVALUE/VECTOR
Fig. 3 Method convergence: almost-singular case.

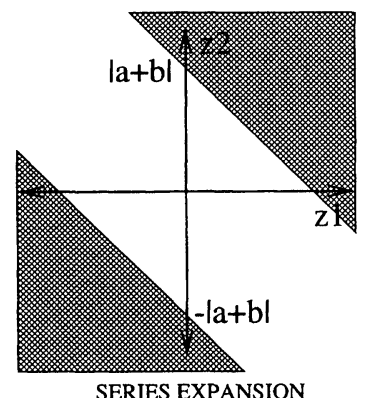

Fig. 4 Method convergence: almost-indistinct case.

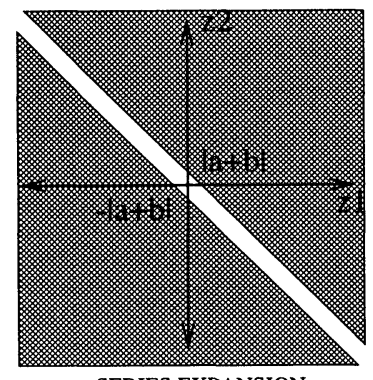

SERIES EXPANSION

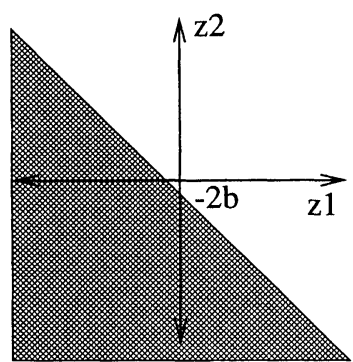

EIGENVALUE/VECTOR
Fig. 5 Method convergence: almost-singular and indistinct cases. 
Table 2 Perturbation method validity

\begin{tabular}{lll}
\hline \hline Eigenvalue & Low damping $^{\mathrm{a}}$ & High damping $^{\mathrm{b}}$ \\
\hline Distant & Eigen approx. $\sqrt{ }$ & Eigen approx. $\sqrt{ }$ \\
& Series method $\times$ & Series method $\sqrt{ }$ \\
Nearby & Eigen approx. $\times$ & Eigen approx. $\times$ \\
& Series method $\times$ & Series method $\sqrt{ }$ \\
\hline \hline
\end{tabular}

${ }^{\mathrm{a}}$ Sensitive to mistuning. ${ }^{\mathrm{b}}$ Not sensitive to mistuning.

a large region with only a few terms by approximating $\lambda_{j}(z)$ in the denominator (matching two similar discontinuous functions). The functional form $1 /\left[i \omega-\lambda_{j}(z)\right]$ yields the required steep nonlinear dependence on mistuning.

In summary, Table 2 follows from Eqs. (24), (25), and the example of series vs eigenvalue/vector convergence. The light-damping limitation of the series method is also noted in Ref. 12. By comparison, the eigenvalue/vector method holds in the lightly damped resonant case, but fails if we have closely spaced modes. Of course, symmetry arguments along with Eqs. (24) and (25) continue to hold. To extend the eigenvalue/vector method to the nearby eigenvalues case requires the tracking of sharply veering eigenvalues and eigenvectors.

\section{Forced Response by Eigenstructure Perturbation}

Our eigenvalue/vector perturbation approach, shown next, is valuable for two reasons: First, it allows for a large region of method validity as in the example in Sec. IV.C, and second, it states the forced response solution in terms of eigenvalues and eigenvectors providing a link to familiar concepts in linear systems. The basic idea is very simple: We write the response in terms of eigenvalues and eigenvectors, and this allows an approximation of the mistuned response by perturbation of the eigenvalues and eigenvectors about the tuned point.

\section{A. Jordan Decomposition}

Forced response $\boldsymbol{X}$ in the frequency domain is given by expression (13):

$$
X(z)=A^{-1}(z) B_{l}(z)
$$

where $A(z)=[i \omega I-M(z)]$. To develop a simple functional form for $X(z)$, we must approximate the inverse matrix $A^{-1}(z)$, which we do by bringing the symmetry tools of Sec. II together with eigenstructure perturbation concepts shown next.

Assume the tuned linearization matrix $\bar{M}=M(0)$ of Eq. (16) has distinct eigenvalues, this is true generically (almost always). ${ }^{9}$ Then, $M(z)$ has a diagonal Jordan form, where the eigenvalues and eigenvectors vary smoothly with $z$ (see Ref. 16):

$$
\begin{aligned}
M(z) & =T(z) \Lambda(z) T^{-1}(z) \\
& =\left[\begin{array}{ccc}
\mid & & \mid \\
V_{1}(z) & \cdots & V_{n}(z) \\
\mid & & \mid
\end{array}\right]\left[\begin{array}{ccc}
\lambda_{1}(z) & & 0 \\
& \ddots & \\
0 & & \lambda_{n}(z)
\end{array}\right]\left[\begin{array}{c}
-U_{1}(z)- \\
\vdots \\
-U_{n}(z)-
\end{array}\right]
\end{aligned}
$$

Here, $\Lambda(z)$ is a diagonal matrix of eigenvalues, and $T(z)$ and $T^{-1}(z)$ are matrices of right and left eigenvectors, respectively. Let the right eigenvectors $\boldsymbol{V}_{j}(z)$ be normalized as $\left\|\boldsymbol{V}_{j}(0)\right\|=1$ for any $j$. By definition, $A(z)=T(z)[i \omega I-\Lambda(z)] T^{-1}(z)$, and so the inverse matrix $A^{-1}(z)$ is given by

$$
A^{-1}(z)=[i \omega I-M(z)]^{-1}=T(z)[i \omega I-\Lambda(z)]^{-1} T^{-1}(z)
$$

where

$$
[i \omega I-\Lambda(z)]^{-1}=\left[\begin{array}{ccc}
\frac{1}{i \omega-\lambda_{1}(z)} & & 0 \\
0 & \ddots & \frac{1}{i \omega-\lambda_{n}(z)}
\end{array}\right]
$$

Expression (35) may be rewritten:

$$
A^{-1}(z)=\sum_{q=1}^{n} \frac{1}{i \omega-\lambda_{q}(z)} \underbrace{\left[\begin{array}{c}
1 \\
\boldsymbol{V}_{q}(z) \\
1
\end{array}\right]\left[-\boldsymbol{U}_{q}(z)-\right]}_{n \times n \text { complex matrix }}
$$

where $n=r m$.

\section{B. Response per Mode with Symmetry}

Now consider response $\boldsymbol{X}^{j, d}(\boldsymbol{z})$ from a single mode $j, d$ (numbering of Theorem 3.1). From Eqs. (33) and (37) we have

$$
\boldsymbol{X}^{j, d}(z)=\frac{\boldsymbol{V}_{j}^{d}(z) \boldsymbol{U}_{j}^{d}(z) \boldsymbol{B}_{l}(z)}{i \omega-\lambda_{j}^{d}(z)}
$$

where the response for all modes is given by

$$
\boldsymbol{X}(z)=\sum_{j=0}^{r-1} \sum_{d=1}^{m} \boldsymbol{X}^{j, d}(z)
$$

Recall that $\boldsymbol{V}_{j}^{d}(\boldsymbol{z}), \boldsymbol{U}_{j}^{d}(\boldsymbol{z})$, and $\boldsymbol{B}_{l}(\boldsymbol{z})$ have the symmetries of Table 1; these imply that

$$
\begin{aligned}
& \boldsymbol{U}_{j}^{d}(z)= {\left[\boldsymbol{u}_{j}^{d}(z), p_{j}^{-1} \boldsymbol{u}_{j}^{d}(\varphi z), p_{j}^{-2} \boldsymbol{u}_{j}^{d}\left(\varphi^{2} z\right), \ldots, p_{j}^{-r+1} \boldsymbol{u}_{j}^{d}\left(\varphi^{r-1} z\right)\right] } \\
& \boldsymbol{V}_{j}^{d}(z)=\left[\begin{array}{c}
\boldsymbol{v}_{j}^{d}(z) \\
p_{j} \boldsymbol{v}_{j}^{d}(\varphi z) \\
p_{j}^{2} \boldsymbol{v}_{j}^{d}\left(\varphi^{2} z\right) \\
\vdots \\
p_{j}^{r-1} \boldsymbol{v}_{j}^{d}\left(\varphi^{r-1} z\right)
\end{array}\right], \quad \boldsymbol{B}_{l}(z)=\left[\begin{array}{c}
\boldsymbol{\beta}(z) \\
p_{l} \boldsymbol{\beta}(\varphi z) \\
p_{l}^{2} \boldsymbol{\beta}\left(\varphi^{2} z\right) \\
\vdots \\
p_{l}^{r-1} \boldsymbol{\beta}\left(\varphi^{r-1} z\right)
\end{array}\right]
\end{aligned}
$$

where $\boldsymbol{v}_{j}^{d}(z), \boldsymbol{u}_{j}^{d}(z)$, and $\boldsymbol{\beta}(\boldsymbol{z})$ correspond to the first (m-sized) block of $\boldsymbol{V}_{j}^{d}(z), \boldsymbol{U}_{j}^{d}(z)$, and $\boldsymbol{B}_{l}(z)$, respectively.

Equation (40) allows us to write the response of the first blade [first block of $\boldsymbol{X}^{j, d}(\boldsymbol{z})$ in Eq. (38)] as

$$
X_{1}^{j, d}(z)=\frac{1}{i \omega-\lambda_{j}^{d}(z)}\left[\sum_{s=0}^{r-1} p_{l-j}^{s} u_{j}^{d}\left(\varphi^{s} z\right) \beta\left(\varphi^{s} z\right)\right] v_{j}^{d}(z)
$$

Equation (41) is a great simplification; it reduces computing the forced response $\boldsymbol{X}(\boldsymbol{z})$ from an $\mathrm{rm}$-sized vector problem to a first-blade $m$-sized problem. Response of the first blade as a result of all modes follows from Eq. (39):

$$
\boldsymbol{X}_{1}(z)=\sum_{j=0}^{r-1} \sum_{d=1}^{m} \boldsymbol{X}_{1}^{j, d}(z)
$$

Subsequent response for all blades is given by symmetry (21):

$$
\boldsymbol{X}(z)=\left[\boldsymbol{X}_{1}(z), p_{l} \boldsymbol{X}_{1}(\varphi z), p_{l}^{2} \boldsymbol{X}_{1}\left(\varphi^{2} z\right), \ldots, p_{l}^{r-1} \boldsymbol{X}_{1}\left(\varphi^{r-1} z\right)\right]
$$

\section{Tuned Response}

When the system is tuned $(z=0)$, Eq. (42) reduces to

$$
\boldsymbol{X}_{1}(0)=r \sum_{d=1}^{m} \frac{\boldsymbol{u}_{L}^{d}(0) \boldsymbol{\beta}(0)}{i \omega-\lambda_{L}^{d}(z)} v_{L}^{d}(0)
$$


where $L=l \bmod r$. For example, if $r=10$ and $l=12$ [meaning $\zeta(\theta)=\sin (12 \theta)$ in Fig. 2], then only $j=L=2$ modes will appear in Eq. (44).

The preceding statements can also be seen geometrically: $y$ $=T^{-1}(z) x$ is the change of coordinates that transforms Eq. (11) into diagonal form:

$$
\dot{\boldsymbol{y}}=\Lambda(z) y+T^{-1}(z) B_{l}(z) e^{i \omega t}
$$

Now, $T^{-1}(z) B_{l}(z)$ is the projection of the forcing vector $\boldsymbol{B}_{l}(z)$ into the diagonal coordinate system. The matrix $T^{-1}(z)$ consists of left eigenvectors $U_{j}^{d}(z)$ of $M(z)$. When the system is tuned, $\boldsymbol{B}_{l}(0)$ lines up with the $L$ th right eigenvector block $V_{L}^{d}(0)$ :

$$
\begin{gathered}
\boldsymbol{V}_{L}^{d}(0)=\left(\boldsymbol{v}, p_{l} \boldsymbol{v}, p_{l}^{2} v, \ldots, p_{l}^{r-1} v\right) \\
\boldsymbol{B}_{l}(0)=\left(\boldsymbol{\beta}, p_{l} \boldsymbol{\beta}, p_{l}^{2} \boldsymbol{\beta}, \ldots, p_{l}^{r-1} \boldsymbol{\beta}\right)
\end{gathered}
$$

More precisely, $\boldsymbol{B}_{l}(0)$ projects onto a subspace defined by the span of $V_{L}^{d}(0)$ as $d$ varies between 1 and $m$. So, the only non- trivial equations in Eq. (45) are those corresponding to the $L$ th modes:

$$
\begin{gathered}
\dot{y}_{j}^{d}=\lambda_{j}^{d}(0) y_{j}^{d}, \quad \text { for } \quad j \neq L \\
\dot{y}_{L}^{d}=\lambda_{L}^{d}(0) y_{L}^{d}+\boldsymbol{U}_{L}^{d}(0) \boldsymbol{B}_{l}(0) e^{i \omega t}
\end{gathered}
$$

As $z$ is varied away from zero, the eigenvectors $V(z)$ and $U(z)$ shift, and the $L$ th right eigenvector subspace drops away from the forcing vector $\boldsymbol{B}_{l}(\boldsymbol{z})$. For the mistuned case, $\boldsymbol{B}_{l}(\boldsymbol{z})$ has a projection onto all eigenvectors, the structure [Eqs. (48) and (49)] fails, and all modes come into play (as in Fig. 6). The amount by which any specific mode-shape $V_{j}^{d}(z)$ appears is determined by the size of projection $\boldsymbol{U}_{j}^{d}(\boldsymbol{z}) \boldsymbol{B}_{l}(\boldsymbol{z})$ scaled by the inverse eigenvalue $\mu_{j}^{d}(z)=1 /\left[i \omega-\lambda_{j}^{d}(z)\right]$, precisely the scalar term $\boldsymbol{U}_{j}^{d}(z) \boldsymbol{B}_{l}(z) /\left[i \omega-\lambda_{j}^{d}(z)\right]$ in Eq. (38). Consequently, lightly damped modes close to resonance will appear far more readily with mistuning than highly damped modes forced far from resonance.

\section{Eigenstructure Approximations}

Up to now, we have not made any approximations, and so the modal expression of Eqs. $(41-43)$ is an exact solution to
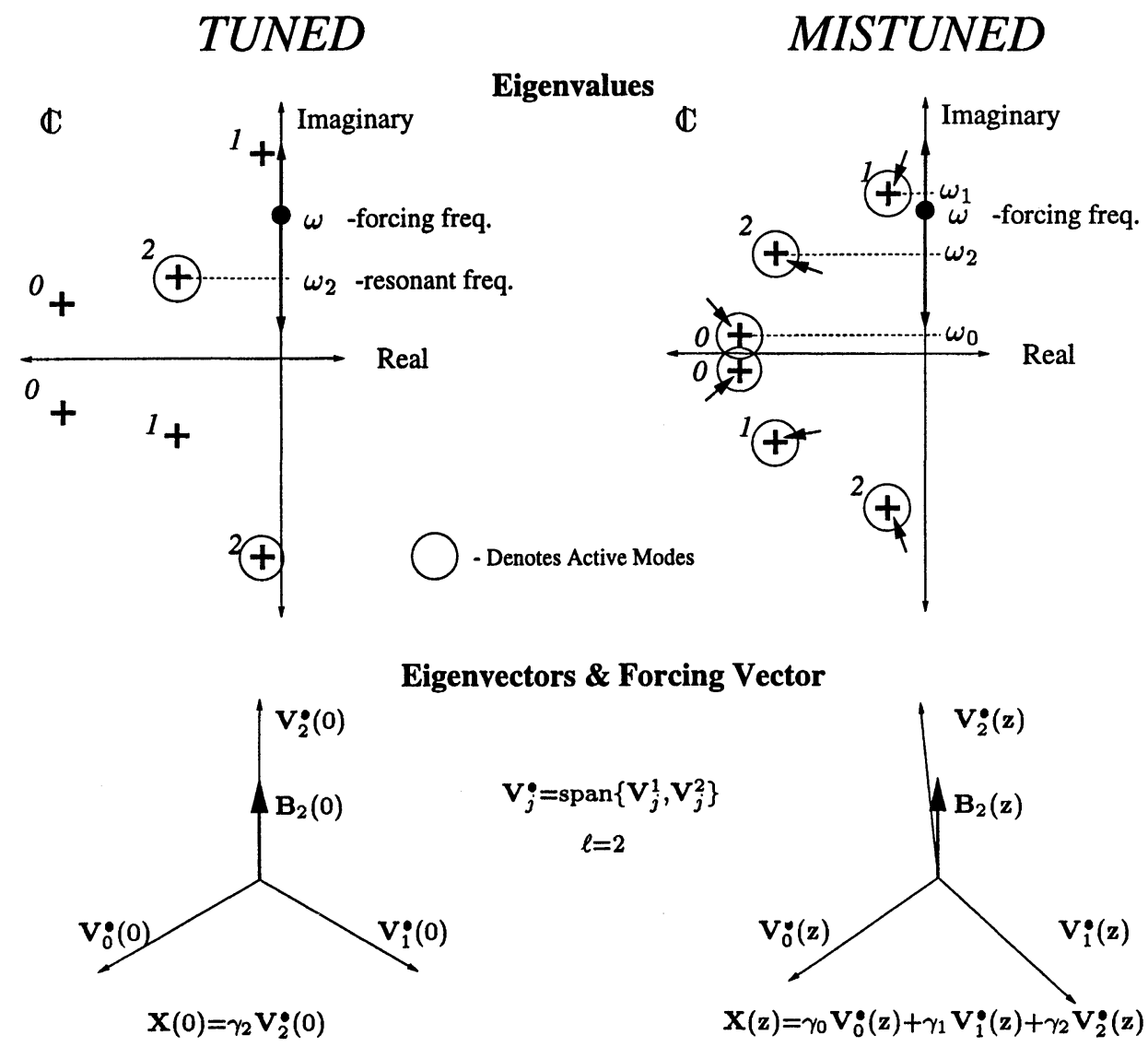

Eigenvectors \& Forcing Vector

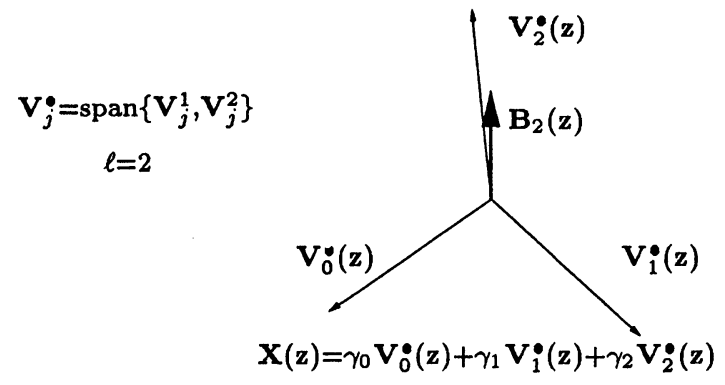

Response: Bode Plots
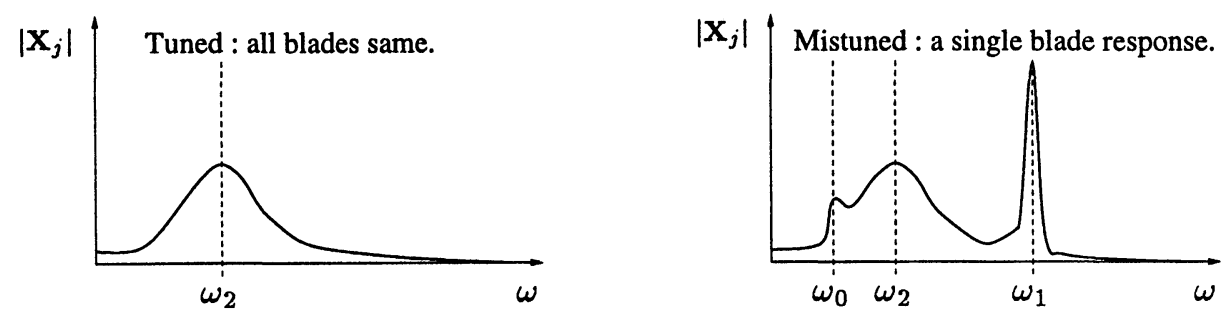

Fig. 6 Geometric explanation of mistuning sensitivity. 
the linear forced response problem, Eq. (13). An obvious firstcut approximation is to drop all highly damped modes. In this case, summation (42) is only taken over $j=l \bmod r$ (the tuned modes), plus any additional mistuned modes $j, d$, which satisfy

$$
\left|\operatorname{Re}\left[\lambda_{j}^{d}(0)\right]\right|<\delta \Rightarrow\left|\mu_{j}^{d}(0)\right|=\frac{1}{\left|i \omega-\lambda_{j}^{d}(0)\right|}>\frac{1}{\delta}
$$

where $1 / \delta$ is the (roughly) desired accuracy. Equation (50) is a useful estimate, yet it does not address our primary goal of expressing Eq. (41) as a simple functional form in $z$. To do so, we turn to standard tools in eigenvalue and eigenvector perturbation.

\section{Inverse Eigenvalue Perturbation}

We need to approximate the term

$$
\mu_{j}^{d}(z)=\frac{1}{i \omega-\lambda_{j}^{d}(z)}
$$

in Eq. (41). Here, $\mu_{j}^{d}(z)$ is an eigenvalue of $A^{-1}(z)$ and $\lambda_{j}^{d}(z)$ is an eigenvalue of $M(z)$. Eigenvalues $\lambda_{j}^{d}(z)$ are approximated by the methods of Ref. 9 . Remember that eigenvalue $\lambda_{j}^{d}(z)$ has symmetry:

$$
\lambda_{j}^{d}(z)=\lambda_{j}^{d}(\varphi z)
$$

and so is invariant under rotation (see Lemma A.5). Invariance under rotation implies

$$
\begin{aligned}
\lambda_{j}^{d}(z)= & \lambda_{j}^{d}(0)+a_{j, d}\left(z_{1}+z_{2}+\cdots+z_{r}\right) \\
& +b_{j, d}\left(z_{1}^{2}+z_{2}^{2}+\cdots+z_{r}^{2}\right) \\
& +c_{1}^{j, d}\left(z_{1} z_{2}+z_{2} z_{3}+\cdots+z_{r} z_{1}\right) \\
& +c_{2}^{j, d}\left(z_{1} z_{3}+z_{2} z_{4}+\cdots+z_{r} z_{2}\right) \\
& \vdots \\
& +c_{k}^{j, d}\left(z_{1} z_{1+k}+z_{2} z_{2+k}+\cdots+z_{r} z_{k}\right)+\mathcal{O}\left(\|z\|^{3}\right) \\
= & \lambda_{j}^{d}(O)+a_{j, d} \sum_{i=1}^{r} z_{i}+z^{T}\left(S_{j, d}\right) z+\mathcal{O}\left(\|z\|^{3}\right)
\end{aligned}
$$

where $k$ is given by

$$
k \triangleq\left\{\begin{array}{ccc}
\frac{r}{2} & r & \text { even } \\
\frac{r-1}{2} & r & \text { odd }
\end{array}\right.
$$

There are some slight differences in notation between Ref. 9 and the current paper. In Ref. 9, coefficients $a, b, c_{1}, \ldots, c_{k}$ are real and describe motion of the real stability increase $s(z)$. Here, coefficients $a, b, c_{1}, \ldots, c_{k}$ are complex to capture complex eigenvalues. ${ }^{9}$

The linear term $a \sum_{i=1}^{r} z_{i}$ does not capture mistuning dependence; it only reacts to the averaged or tuned $\sum_{i=1}^{r} z_{i}$ portion. It is the quadratic term $z^{T} S z$, which really reveals eigenvalue motion as a function of mistuning and captures motion caused by zero average mistuning: $\sum_{i=1}^{r} z_{i}=0$. Since $\mu(z)$ in Eq. (51) can depend strongly on $\lambda(z)$, it is crucial to capture this second-order eigenvalue dependence on mistuning given by $z^{T} S z$.

Differentiating Eq. (52) shows that coefficients $a, b, c_{1}, \ldots$, $c_{k}$ correspond to eigenvalue derivatives:

$$
a_{j, d}=\frac{\partial \lambda_{j}^{d}}{\partial z_{1}}(0), \quad b_{j, d}=\frac{1}{2} \frac{\partial^{2} \lambda_{j}^{d}}{\partial z_{1}^{2}}(0), \quad c_{i}^{j, d}=\frac{\partial^{2} \lambda_{j}^{d}}{\partial z_{1} \partial z_{1+i}}(0)
$$

These eigenvalue derivatives with respect to parameters are computed as in Ref. 9. This yields an approximation for the $1 /\left[i \omega-\lambda_{j}^{d}(z)\right]$ term in Eq. (41) by approximating eigenvalue $\lambda_{j}^{d}(z)$ in the denominator of Eq. (51).

\section{Coupling Approximation}

Our next goal is to approximate the remainder of expression (41), namely, find an approximation for the coupling term:

$$
\Psi_{j}^{d}(z)=\left[\sum_{s=0}^{r-1} p_{l-j}^{s} \boldsymbol{u}_{j}^{d}\left(\varphi^{s} z\right) \boldsymbol{\beta}\left(\varphi^{s} z\right)\right] \boldsymbol{v}_{j}^{d}(z)
$$

Consequently, Eq. (41) may be rewritten as $\boldsymbol{X}_{1}^{j, d}(z)=$ $\mu_{j}^{d}(z) \Psi_{j}^{d}(z)$. The term $\Psi$ varies slowly with $z$; eigenvectors change slowly unless eigenvalues are clustered because rate equations such as Eq. (58) always depend on eigenvalue distance $\lambda_{p}-\lambda_{p}$ in the denominator. Consequently, it is acceptable to approximate $\Psi(z)$ as a first-order function in $z$.

Equation (55) can be further split into two parts: the summation

$$
g_{j}^{d}(z)=\sum_{s=0}^{r-1} p_{l-j}^{s} \boldsymbol{u}_{j}^{d}\left(\varphi^{s} z\right) \boldsymbol{\beta}\left(\varphi^{s} z\right)
$$

and the first block $\boldsymbol{v}_{j}^{d}(z)$ of right eigenvector $\boldsymbol{V}_{j}^{d}(z)$. So, $\Psi_{j}^{d}(z)$ $=g_{j}^{d}(z) v_{j}^{d}(z)$, where $g_{j}^{d}(z)$ is a scalar function and $\boldsymbol{v}_{j}^{d}(z) \in \mathbb{C}^{m}$.

a. Right eigenvector perturbation. We linearly approximate $v_{j}^{d}(z)$ found in Eq. (55) by

$$
\begin{aligned}
& \boldsymbol{v}_{j}^{d}(\boldsymbol{z}) \approx\left[\boldsymbol{v}_{j}^{d}(0)\right]+\left[\frac{\partial \boldsymbol{v}_{j}^{d}}{\partial z_{1}}(0)\right] z_{1}+\left[\frac{\partial \boldsymbol{v}_{j}^{d}}{\partial z_{2}}(0)\right] z_{2} \\
&+\cdots+\left[\frac{\partial \boldsymbol{v}_{j}^{d}}{\partial z_{r}}(0)\right] z_{r}
\end{aligned}
$$

Because $\boldsymbol{v}_{j}^{d}(0)$ corresponds to the first block of tuned right eigenvector $V_{j}^{d}(0)$, it may be computed from Theorem 3.1. The required eigenvector derivatives are given by formulas similar to those in Ref. 16:

$$
\frac{\partial \boldsymbol{v}_{j}^{d}}{\partial z_{i}}(0)=\sum_{p, s \neq j, d}\left\{\frac{\boldsymbol{U}_{p}^{s}(0)\left[\frac{\partial M}{\partial z_{i}}(0)\right] \boldsymbol{V}_{j}^{d}(0)}{\lambda_{j}^{d}(0)-\lambda_{p}^{s}(0)}\right\} \boldsymbol{v}_{p}^{s}(0)
$$

As in the eigenvalue perturbation, all we need to do is compute the tuned eigenvalues and right, left eigenvectors, $\lambda(0), \boldsymbol{U}(0)$, and $\boldsymbol{V}(0)$, which substitute into Eqs. (57) and (58) to yield a linear approximation for $\boldsymbol{v}_{j}^{d}(\boldsymbol{z})$. If desired, one may make further approximation by dropping from summation (58) any mode $p, s$, which is far from target mode $j, d, \mid \lambda_{j}^{d}(0)-$ $\lambda_{p}^{s}(0) \mid$ large.

b. Summation approximation. It remains to approximate the summation term $g_{j}^{d}(z)$ of Eq. (56). It is easy to show that $g_{j}^{d}(z)$ has symmetry:

$$
g_{j}^{d}(\varphi z)=p_{j-\iota} g_{j}^{d}(z)
$$

Symmetry (59) implies that $g_{j}^{d}(z)$ has the form

$$
\begin{aligned}
& g_{j}^{d}(z)=g_{j}^{d}(0)+\bar{g}_{j}^{d}\left(z_{1}+p_{l-j} z_{2}+p_{l-j}^{2} z_{3}+\cdots+p_{l-j}^{r-1} z_{r}\right) \\
& +\mathcal{O}\left(\|z\|^{2}\right)
\end{aligned}
$$

To complete a first-order approximation of $g_{j}^{d}(z)$, we need to find the complex coefficients $g_{j}^{d}(0)$ and $\bar{g}_{j}^{d}$. The tuned portion $g_{j}^{d}(0)$ is computed from Eq. (56) and Theorem 3.1:

$$
\begin{gathered}
g_{j}^{d}(0)=0 \text { for } j \neq L \\
g_{L}^{d}(0)=r \boldsymbol{u}_{L}^{d}(0) \boldsymbol{\beta}(0)
\end{gathered}
$$


Coefficient $\bar{g}_{j}^{d}$ is obtained by differentiating Eqs. (56) and (60) with respect to $z_{1}$ :

$$
\begin{aligned}
\bar{g}_{j}^{d} & =\frac{\partial g_{j}^{d}}{\partial z_{1}}(0)=\sum_{s=0}^{r-1} p_{l-j}^{s}\left\{\left[\frac{\partial \boldsymbol{u}_{j}^{d}}{\partial z_{1-s}}(0)\right][\boldsymbol{\beta}(0)]\right. \\
& \left.+\left[\boldsymbol{u}_{j}^{d}(0)\right]\left[\frac{\partial \boldsymbol{\beta}}{\partial z_{1-s}}(0)\right]\right\}
\end{aligned}
$$

It remains to find left eigenvector derivatives with respect to parameters, $\partial u / \partial z_{i}$, and as in the right eigenvector case, this is done by standard methods as in Ref. 16. Analogous to Eq. (58), we have

$$
\frac{\partial \boldsymbol{u}_{j}^{d}}{\partial z_{i}}(0)=\sum_{p, s \neq j, d}\left\{\frac{\boldsymbol{U}_{j}^{d}(0)\left[\frac{\partial M}{\partial z_{i}}(0)\right] \boldsymbol{V}_{p}^{s}(0)}{\lambda_{j}^{d}(0)-\lambda_{p}^{s}(0)}\right\} \boldsymbol{u}_{p}^{s}(0)
$$

and once more we only need to find the tuned eigenvalue and left, right eigenvectors, $\lambda(0), U(0)$, and $V(0)$, from Theorem 3.1 to complete the calculation.

\section{Combining Terms}

Combining linear approximations for $\boldsymbol{v}_{j}^{d}(z)$ [Eqs. (57) and (59)] and $g_{j}^{d}(z)$ [Eqs. (60-63)] yields a linear approximation for the coupling term $\Psi_{j}^{d}(z)$ of Eq. (55):

$$
\begin{aligned}
& \Psi_{j}^{d}(z)=g_{j}^{d}(z) v_{j}^{d}(z)=\boldsymbol{\alpha}_{0}^{j, d}+\boldsymbol{\alpha}_{1}^{j, d} z_{1}+\boldsymbol{\alpha}_{2}^{j, d} z_{2}+\cdots+\boldsymbol{\alpha}_{r}^{j, d} z_{r} \\
& \quad+\mathcal{O}\left(\|z\|^{2}\right)
\end{aligned}
$$

where $\boldsymbol{\alpha}_{i}^{j, d}$ are known, constant, complex, $m$-sized, column vectors. Recall that the forced response of blade one, $\boldsymbol{X}_{1}^{j, d}(\boldsymbol{z})$, because of the $j, d$ th mode is taken from Eqs. (41) and (55):

$$
X_{1}^{j, d}(z)=\frac{\Psi_{j}^{d}(z)}{i \omega-\lambda_{j}^{d}(z)}
$$

Here inverse eigenvalue $1 /\left[i \omega-\lambda_{j}^{d}(z)\right]$ of Eq. (51) is accurately captured by approximation (52) of $\lambda_{j}^{d}(z)$ in the denominator. Approximation (64) together with the denominator approximation of eigenvalue $\lambda_{j}^{d}(z)$ as shown earlier yields the approximate forced response of blade one from mode $j, d$ :

$$
\boldsymbol{X}_{1}^{j, d}(z) \approx \frac{\boldsymbol{\alpha}_{0}^{j, d}+\boldsymbol{\alpha}_{1}^{j, d} z_{1}+\boldsymbol{\alpha}_{2}^{j, d} z_{2}+\cdots+\boldsymbol{\alpha}_{r}^{j, d} z_{r}}{i \omega-\lambda_{j}^{d}(0)-a_{j, d} \sum_{i=1}^{r} z_{i}-z^{T}\left(S_{j, d}\right) z}
$$

In cleaner notation (drop all $j, d$ indexing), the approximate response of blade one caused by a single mode is given by

$$
\boldsymbol{X}_{1}^{j, d}(z) \approx \frac{\boldsymbol{\alpha}_{0}+\boldsymbol{\alpha}_{1} z_{1}+\boldsymbol{\alpha}_{2} z_{2}+\cdots+\boldsymbol{\alpha}_{r} z_{r}}{i \omega-\lambda(0)-a \sum_{i=1}^{r} z_{i}-z^{T} S z}
$$

Here $\boldsymbol{\alpha}_{i}$ are known, constant, $m$-sized vectors; $\omega=l \Omega$ is the forcing frequency; $\lambda(0)$ is an eigenvalue of tuned linearization matrix $M(0) ; a$ is a known complex coefficient; and $S$ is a known complex matrix containing coefficients $b, c_{1}, \ldots, c_{k}$ as in Eq. (52). Further, it follows from Eq. (61) that $\boldsymbol{\alpha}_{0}^{j, d}=0$ if $j$ $\neq l$ mod $r$ since $\boldsymbol{\alpha}_{0}^{j, d}$ corresponds to the tuned portion of the response. An approximation for the total response of blade one follows from Eqs. (42) and (66):

$$
\begin{aligned}
\boldsymbol{X}_{1}(z) & =\sum_{j=0}^{r-1} \sum_{d=1}^{m} \boldsymbol{X}_{1}^{j, d}(z) \\
\approx & \sum_{j=0}^{r-1} \sum_{d=1}^{m} \frac{\boldsymbol{\alpha}_{0}^{j, d}+\boldsymbol{\alpha}_{1}^{j, d} z_{1}+\boldsymbol{\alpha}_{2}^{j, d} z_{2}+\cdots+\boldsymbol{\alpha}_{r}^{j, d} z_{r}}{i \omega-\lambda_{j}^{d}(0)-a_{j, d} \sum_{i=1}^{r} z_{i}-z^{T}\left(S_{j, d}\right) z}
\end{aligned}
$$

In addition, we can drop terms in Eq. (68), which correspond to highly damped modes. In that case the summation is taken over all tuned modes with $j=l$ mod $r$ plus those that satisfy Eq. (50). Finally, the response for all blades is given by symmetry (43).

Our analysis goal has now been achieved. Equations (43), (66), and (68) provide a simple functional form in $z$ for forced response $\boldsymbol{X}(\boldsymbol{z})$. Once we have computed coefficients $\boldsymbol{\alpha}_{i}, a$, and $b, c_{1}, \ldots, c_{k}$ of matrix $S$ for all desired modes, we know the forced response for arbitrary mistuning. More importantly, functional forms (43), (66), and (68) allow for an intuitive understanding of the mode localization problem and permit sensitivity or tradeoff studies as in Sec. VII.

\section{E. Mistuning Sensitivity}

There exists a simple and elegant geometric interpretation of the mode localization solution. Consider a three-blade, twostates-per-blade example as in Fig. 6.

First consider the tuned case. Tuned matrix $M(0)$ of Eq. (11) has six eigenvalues $\lambda_{j}^{d}(0)$. These eigenvalues are labeled by index $j$ and plotted as crosses in the complex plane at the topleft of Fig. 6. Each tuned eigenvalue $\lambda_{j}^{d}(0)$ has associated tuned right eigenvectors $\boldsymbol{V}_{j}^{d}(0)$ with constant inter-blade-phase-angle $2 \pi j / r, j=0,1$, or 2 . Theorem 3.1 implies that $\lambda_{j}^{d}$ has complex conjugate $\lambda_{r-j}^{d}$, and so the numbering of modes in Fig. 6 is correct. Our forcing is taken to be purely second modal $[l=2$ in Eq. (11)], and so, only modes with $j=2$ are active (as denoted by circled eigenvalues in the top-left of Fig. 6). In fact, this is a consequence of Eqs. (48) and (49), which state that the tuned forcing vector $B_{l}(0)$ lies in the subspace $V_{L}^{*}(0)$ generated by $\boldsymbol{V}_{L}^{1}(0), \boldsymbol{V}_{L}^{2}(0), \ldots, \boldsymbol{V}_{L}^{m}(0)$. This is denoted schematically in the middle-left of Fig. 6 , where $\boldsymbol{B}_{2}(0)$ exactly lines up with subspace $\boldsymbol{V}_{2}^{*}(0)$. Hence, forcing $\boldsymbol{B}_{2}(0)$ can only affect $j=2$ modes, and so we write in shorthand

$$
\boldsymbol{X}(0)=\gamma_{2} \boldsymbol{V}_{2}(0)
$$

meaning that the response only contains $j=2$ modes. Coefficients in $\gamma_{2}$ are proportional to the inverse of distance $\mid \lambda_{j}^{d}(0)$ $-i \omega \mid$, thus, lightly damped modes forced near resonance create large $\gamma$. In our example, $\lambda_{2}^{1}(0)$ has medium damping (mode $j=2, d=1$ in top-left of the complex plane), whereas the lightly damped mode $\lambda_{2}^{2}(0)$ (mode $j=2, d=2$ at the bottomleft of Fig. 6) is far from resonance for all positive rotation speeds of the fan. Hence, $j=2$ modes generate the gentle Bode plot at the bottom-left of Fig. 6, with associated resonant frequency $w_{2}=\operatorname{Im}\left[\lambda_{2}^{1}(0)\right]$.

Now assume we allow mistuning. First of all, the eigenvalues shift as shown in the top-right of Fig. 6 and this movement is captured by expansion (52). Such motion may be either stabilizing or destabilizing. In our example we show the stabilizing case (least stable eigenvalues move left), as is often the case. Yet the eigenvalues are not perturbed alone, the eigenvectors also shift away from the tuned case as in Eqs. (57) and (58), and as shown pictorially in the middle-right of Fig. 6. Now $\boldsymbol{B}_{2}(z)$ no longer lines up with subspace $\boldsymbol{V}_{2}^{*}(z)$, and so, $\boldsymbol{B}_{2}(z)$ can project onto all the eigenvectors. Notice that $\boldsymbol{B}_{2}(z)$ typically retains its direction because $l=2$ basically corresponds to second modal forcing, as could be caused by two upstream struts, and will not be affected by mistuning of blades. All modes are now excited and this is denoted by all modes being circled in the top-right of Fig. 6 . The associated forced response has the form

$$
X(z)=\gamma_{0} V_{0}^{*}(z)+\gamma_{1} V_{i}^{*}(z)+\gamma_{2} V_{2}^{*}(z)
$$

where the size of coefficients in $\gamma_{j}$ is determined by the amount $\boldsymbol{B}_{2}(z)$ projects onto eigenvector subspace $\boldsymbol{V}_{j}^{*}(z)$ times the inverse of distance $\left|\lambda_{j}^{d}(z)-i \omega\right|$. Because mode one, $\lambda_{1}^{\prime}(z)$, has very light damping, $\gamma_{1}$ will be large at resonance, even for very small shifts in the eigenvectors. As a result we get the 
Bode plot shown in the bottom right of Fig. 6 with the severe peak at $\omega_{1}=\operatorname{Im}\left[\lambda_{1}^{1}(z)\right]$. In summary, this is the mode localization phenomena. Even when mistuning is stabilizing (modes shift to the left), the appearance and resonance of previously unexcited low-damped modes can cause huge peaks. Moreover, the problem is incredibly sensitive. Tiny changes in the eigenvectors, as created by small random mistuning resulting from machining imperfections, may create unacceptably large vibrations as a result of low damping found in turbomachines. Of course this extends to the case of multiple mode forcing [many values of $l$ in Eq. (11)]. For example, we may have forcing with weak $l=1$ and strong $l=2$ spatial decomposition, creating an acceptable tuned response in modes $j=1$ and $j=$ 2. Upon mistuning, the large $l=2$ forcing component will excite an unacceptable response in the lightly damped $j=1$ mode.

\section{F. Two Misconceptions}

At this point we can dispel two misconceptions found in the literature.

1) Sensitivity to mistuning is caused primarily by light damping and the destruction of eigenvector symmetry (see Fig. 6), not mode-veering or weak/strong blade coupling (except if these cause or combine with light damping) [see Eqs. (38) and (39)]. These equations are exact. Weak ḅlade coupling causes eigenvalues to cluster and creates fast eigenvalue/vector motion [veering of $\lambda(z), V(z)$, and $U(z)$ ]. However, this veering occurs in the vicinity of the clustered eigenvalues/vectors; it is confined to a small region. If the clustered eigenvalues are far into the left-half-plane (strong-damping), the net effect on response (38) and (39) is small. Mode-veering without light damping cannot cause mistuning sensitivity. Light damping without mode-veering can cause mistuning sensitivity (see Table 2).

2) There are no bifurcations in the linear forced response problem [Eq. (13)]. Some past researchers ${ }^{17}$ have confused the quickly varying behavior of $\boldsymbol{X}(z)$ caused by the fast, but smooth, dependence on mistuning in Eq. (51) with a strict definition of bifurcations. To be precise, $\hat{z}$ is a bifurcation point for dynamics (13) if $\boldsymbol{X}$ is not smooth at $\hat{z}$. Yes, eigenvalue/ vector motion can be discontinuous. However, $\partial \boldsymbol{X}(\hat{z}) / \partial z$ is always well defined unless an eigenvalue of $M(\hat{z})$ lands on the imaginary axis (see Theorem A.2). When this happens, stability is lost; either the eigenvalue continues on into the right half-plane (generic case) or stability is determined by the nonlinear terms we neglected in Sec. III.A. Either way, Eq. (13) is meaningless when $M(\hat{z})$ has an eigenvalue on the imaginary axis. Thus, it is meaningless to study the bifurcations of Eq. (13); when a bifurcation exists, Eq. (13) no longer applies.

\section{G. Summary of Section V}

To close this section we present a short summary of the entire analysis method.

1) Start with any nonlinear model (1), which necessarily has symmetry (5).

2) Select an appropriate equilibrium point $\boldsymbol{x}^{*}(z)$ that has symmetry of Lemma A.2 (see Sec. III.A. for discussion).

3) Compute linearization matrix $M(z)$ and forcing vector $\boldsymbol{B}_{l}(z)$ of Eq. (11) about chosen equilibrium $\boldsymbol{x}^{*}(z)$ (see Sec. III.B). May consider one forcing mode $l$ at a time by linearity.

4) Check stability, as in Ref. 9, to ensure that forced response is meaningful. Transforming to frequency domain yields the linear forced response problem [Eq. (13)].

5) Solve for eigenvalues, $\lambda(0)$, and right, left eigenvectors, $V(0), U(0)$, of tuned matrix $\bar{M}=M(0)$ in Eq. (16) by Theorem 3.1 .

6) Use $\lambda(0)$ and $V(0)$ as in Ref. 9 to compute eigenvalue perturbation for $\lambda(z)$ of Eq. (52). This approximates eigenvalues $\mu(z)$ of inverse matrix $A^{-1}(z)=[i \omega I-M(z)]^{-1}$ in the denominator through expression (51). Further substitute values of $\lambda(0), \boldsymbol{U}(0)$, and $\boldsymbol{V}(0)$ into Eqs. (58) and (63) to compute
Eqs. (57), (60), and (62), which together yield $\Psi(z)$ of Eq. (64). Combine the $\Psi(z)$ of Eq. (64) and the $\mu(z)$ of Eq. (51) to find first blade approximation (65) and (66) for any given mode $j, d$.

7) Sum over tuned modes, $j=l \bmod r$, and any other modes whose amplitude is larger than desired accuracy $1 / \delta$, see Eq. (50), to yield an apprximation of first blade forced response (68).

8) Response for all other blades follows from symmetry (43).

9) Redo the previous step for all relevant spatial modes $l$ of item 3. Summation over all such $l$ modes yields a final result.

Notice that all of the preceding steps only require information at the tuned point $z=0$. The MATLAB code that performs these computations is available by request from the author.

\section{Application to Quasisteady Model}

Our perturbation method is applied to a quasisteady, inviscid, incompressible, linear model (as in Ref. 18), with 11 blades $(r=11)$ and three states per blade $(m=3)$. We choose model parameters that create a lightly damped system. The resulting tuned lightly damped eigenvalues $\lambda_{j}^{d}(0)$ are shown in Fig. 7, labeled by index $j$. Eigenvalues $\lambda_{2}^{3}$ and $\lambda_{9}^{3}$ are the most lightly damped with damping ratio $-\operatorname{Re}[\lambda] /|\lambda|=0.0000330 /$ $0.0376=0.000875$. (All quantities are nondimensional.)

From the tuned eigenvalues of Fig. 7 , we can predict the nature of the tuned and mistuned response. We pick spatial forcing mode $l=1$. Because the tuned system only displays forced modes, only modes $\lambda_{1}^{d}(0)$ are active. From Fig. 7 we see that for positive forcing frequencies all of the $j=1$ modes $\left(\lambda_{1}^{1}, \lambda_{1}^{2}\right.$, and $\left.\lambda_{1}^{3}\right)$ are far from resonance. [Mode $\lambda_{1}^{1}(0)$ has very high damping and is off the scale to the left of Fig. 7. Mode $\lambda_{1}^{2}(0)$ has positive frequency and medium damping. Finally, mode $\lambda_{1}^{3}(0)$ has small damping but negative frequency; thus, it is not excited by the positive forcing frequency.] As a result,

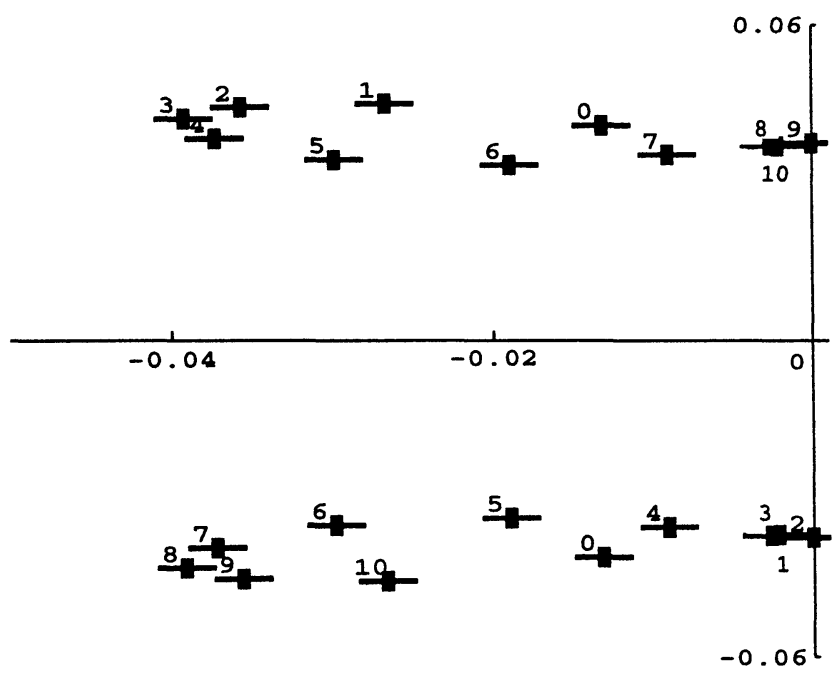

Fig. 7 Tuned eigenvalues $\lambda_{j}^{d}(0)$ labeled by index $j$.

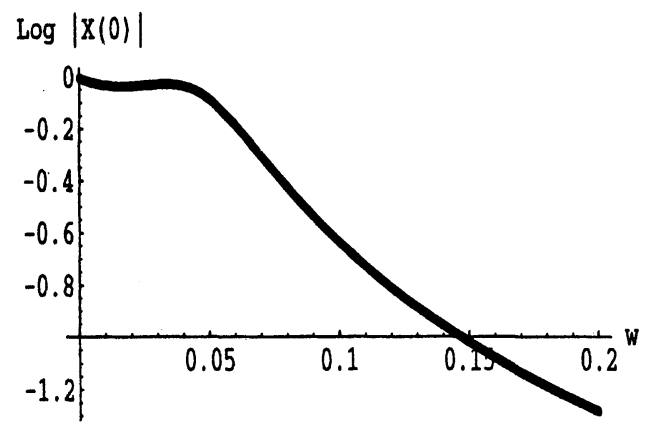

Fig. 8 Tuned Bode plot: $z=0$. 
a) -1.25

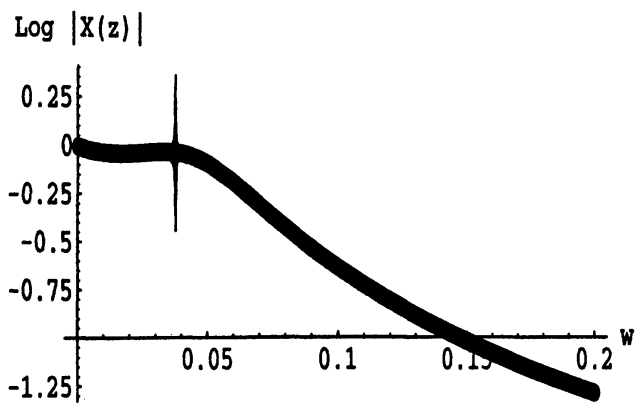

b)

$\log |x(z)|$

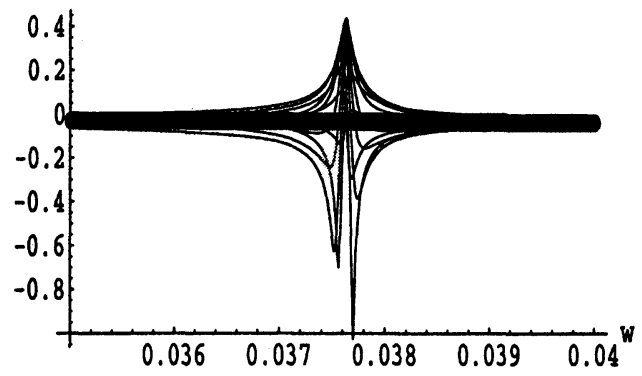

Fig. 9 a) Mistuned Bode plot and b) plot with zoom: $z=z_{\text {rnd. }}$.
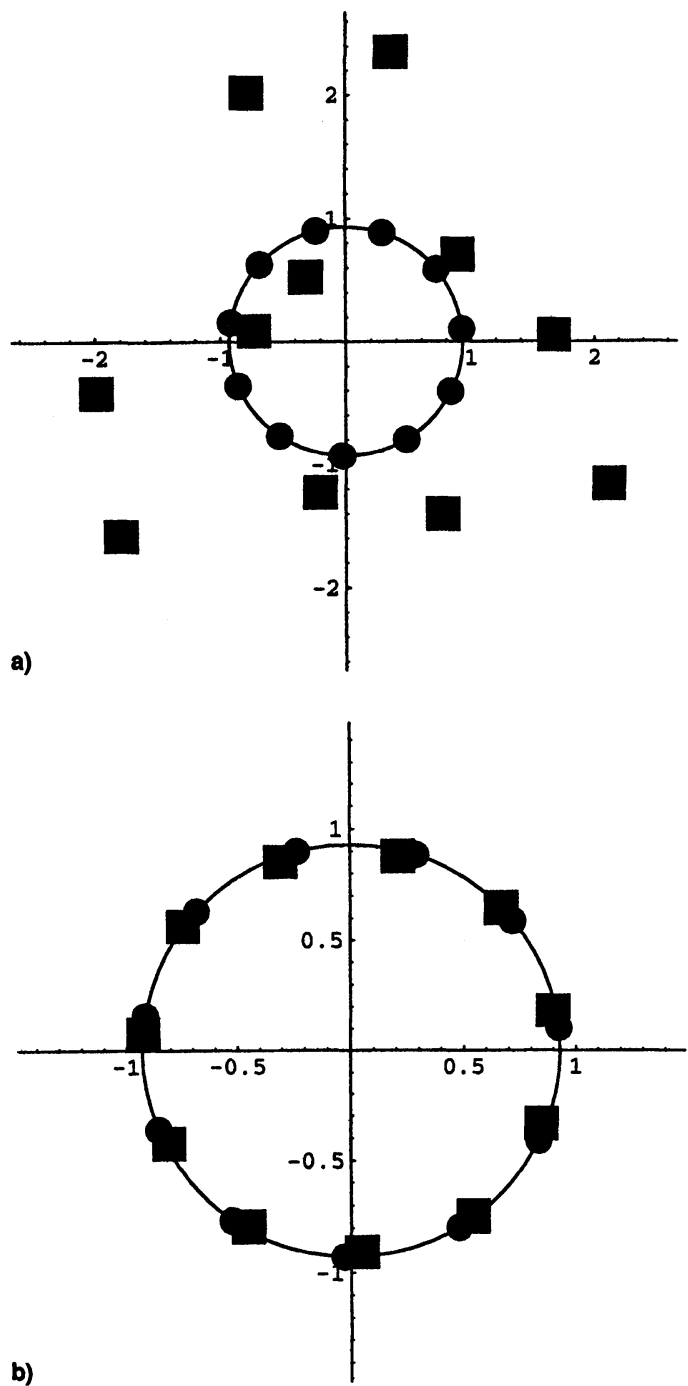

Fig. 10 Tuned vs mistuned mode shapes: $z=0$ and $z=z_{\text {rnd. }}$ Forcing frequency $w=$ a) 0.0376 and b) 0.02 . if we plot the amplitude of vibration as a function of forcing frequency $\omega$, we derive the gentle Bode plot of Fig. 8 with resonant peak $\operatorname{Im}\left[\lambda_{1}^{2}(0)\right]=0.0454$ [see Eqs. (38) and (39)].

Such gentle behavior is lost with the introduction of mistuning. To illustrate this point, we choose a small random mistuning $z_{\text {rnd }}$, where we change the stiffness of each blade by less than $2 \%$. The system is still stable. Plotting the amplitude of response for each blade as a function of forcing frequency $\omega$ yields the Bode plot of Fig. 9. The thick line denotes the tuned response, whereas the 11 thin lines denote the mistuned response of each blade. As demonstrated in Fig. 6, all modes now come into play and the response is dominated by the least stable mode $\lambda_{9}^{3}\left(z_{r n d}\right)$, which resonates at $\omega=0.0376$. We can see that the mistuned response is very sharp and its peak is half an order of magnitude above the tuned response, a direct consequence of the lightly damped $j=9$ eigenvalue: $\operatorname{Re}\left[\lambda_{9}^{3}\left(z_{r n d}\right)\right]=-0.000050$. This demonstrates numerically the sharp sensitivity to mistuning, discussed in Sec. IV. Clearly, the performance is disastrous at $\lambda_{9}^{3}$ resonance: $\omega=0.0376$.

Let us compare the tuned and mistuned response at two frequencies: the resonant frequency $\omega=0.0376$ and a frequency $\omega=0.02$, which is far from resonance. Figure 10 shows the response for the tuned and mistuned system at these frequencies. In both cases, solid circles denote the amplitude
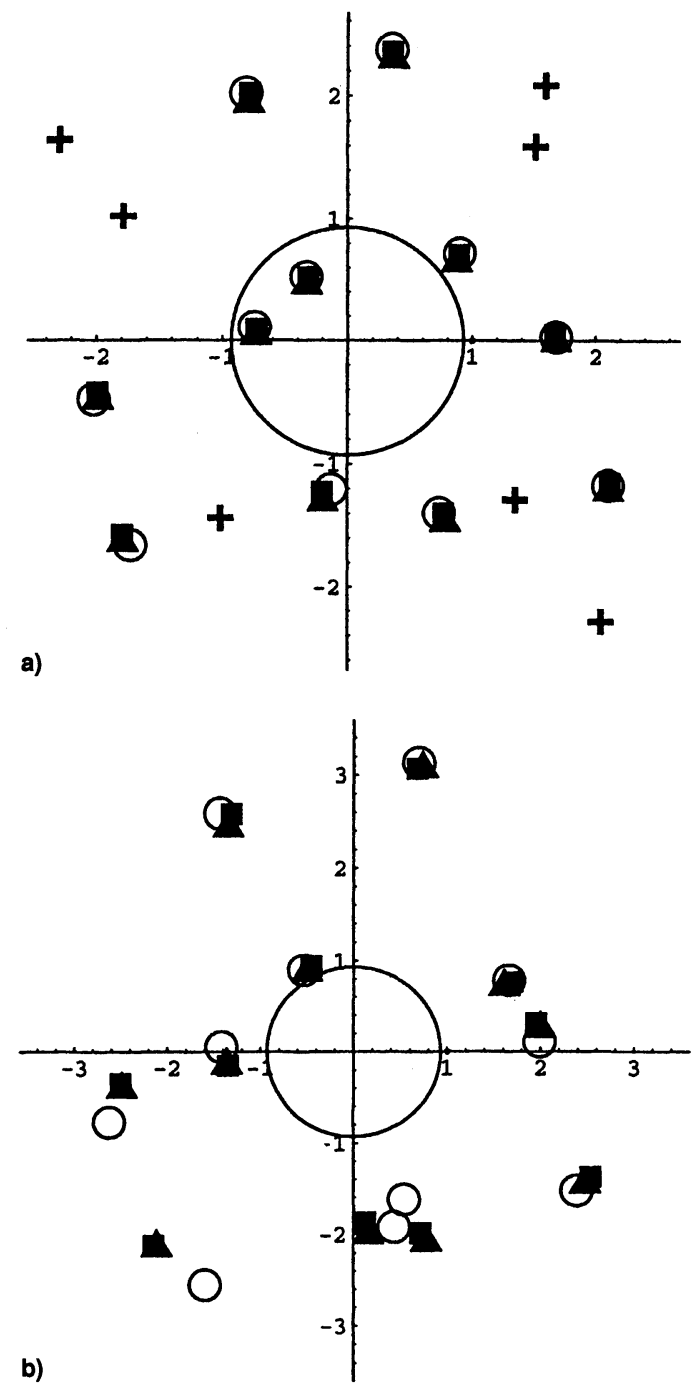

Fig. 11 Approximation comparison at resonance frequency $\omega=$ 0.0376 for small and large mistuning. Exact response of Eq. (13) $=0$, approximate response of Eq. $(68)=\pi$, partial-mode approximate response of Eq. (50) $=\Delta$, series method of Ref. $13=+. z=$ a) $z_{\text {rnd }}$ and b) $5 \times z_{\text {rnd }}$. 
and phase of each blade for the tuned response (all blades have equal ampitude and are separated by a constant-inter-bladephase-angle), wheres solid squares denote the mistuned response. For the resonant frequency, we see that even a small amount of mistuning $z=z_{\text {rnd }}$ can cause a large degree of scatter because of the excitation of previously dormant $j=9$ modes. In contrast, for $\omega=0.02$, far from resonance, other modes are basically not excited and the mistuned response is nearly identical to the tuned response.

All of the preceding data are given by approximation (68). Figure 11 compares the approximation (68) with the exact solution of Eq. (13) for $z=z_{\text {rnd }}$ and $z=5 z_{\text {rnd }}$ at $\omega=0.0376$. As in Fig. 10, we are plotting the amplitude and phase of each blade. Here, the circle at the origin denotes the size of the tuned response; open circles denote the exact mistuned response found by solving Eq. (13) at $z=z_{\text {rnd }}$ or $z=5 z_{\text {rnd }}$ with $\omega=0.0376$; crosses represent the series approximation of Ref. 13 ; solid squares are given by the full-mode approximation (68) and, solid triangles represent a partial-mode approximation with $\delta=0.005$ in Eq. (50).

Our method gives excellent results for small mistuning $z=$ $z_{\text {rnd }}$ and provides acceptable results for larger (roughly 9\%) mistuning $z=5 z_{\text {rnd }}$. In contrast, the series method results have nothing to do with the mistuned forced response. In the small mistuning case, $z=z_{\text {rnd }}$, they are completely inaccurate, whereas in the larger case, $z=5 z_{\text {rnd }}$, they are off the scale of the plot. The series convergence criteria $\rho\left(A_{0}^{-1} \Delta A_{z}\right)<1$ Sec. IV fails in both cases. Consequently, inclusion of additional terms in the series expansion will be of no use.

\section{Engineering Tradeoffs}

Engineering design tradeoffs are summarized next. These tradeoffs are based on symmetry arguments (Sec. II) and hold for any model. Figure 12 shows a schematic of the mistuning space $\mathbb{R}^{r}$ for a fixed forcing frequency $\omega$ : light-gray regions correspond to an acceptable forced response, medium-gray denotes unacceptable response, and the black regions show instability. (We shall prove this figure shortly.)

There is a small region of acceptable response about the origin. Blades are produced within some manufacturing tolerance (dotted white circle). For simplicity, let us assume blades are distributed uniformly within this manufacturing region. In Fig. 12, there is a sliver of unacceptable response inside the manufacturing tolerance region. Hence, the probability of unacceptable response is given by

probability of failure

$$
=\frac{\text { area of unacceptable sliver inside tolerance }}{\text { area of manufacturing tolerance }}
$$

This probability increases if 1) manufacturing tolerance increases; 2) damping decreases, leading to a smaller acceptable response region; and 3) allowable blade amplitude decreases, again creating a smaller acceptable region.

Basically, there exist competing tradeoffs. Engine manufacturers cannot decrease cost (loosen manufacturing tolerances) and increase performance (decrease damping) and lengthen blade life (lower blade response amplitudes). In fact, if we fix the allowable failure probability, there exists a linear constraint between tolerance, damping, and vibration amplitude:

$$
\begin{aligned}
K & \times \text { manufacturing tolerance } \leq \text { damping } \\
& \times \text { allowable blade response }
\end{aligned}
$$

( $K$ is a computable constant). Hence, as companies attempt to reduce cost, prolong engine life, and increase performance for next-generation engines, they will encounter additional mistuning problems.

Luckily, mistuning itself may be used to combat mistuning sensitivity. If we introduce the intentional mistuning $z_{\text {int }}$, shown in Fig. 12, we can guarantee acceptable response under the same manufacturing tolerance. Basically, mistuning $z_{\text {int }}$ creates robust damping.

To demonstrate the preceding tradeoffs, we use the specific model of Sec. VI. However, these tradeoffs are based on symmetry arguments and hold in general. Suppose our conceptual factory produces blades whose stiffnesses vary uniformly between $0.98 k_{0}$ and $1.02 k_{0}$. Blade life is deemed acceptable if blades vibrate less than 10 times the tuned amount: $\|X(z)\| \leq$ $10\|\boldsymbol{X}(0)\|$. We consider the resonance forcing frequency $\omega=$ 0.0376 (see Fig. 9). By Monte Carlo simulation we find that there is a $9.5 \%$ probability of instability and a $4.6 \%$ probability of unacceptable response. This means that both the black unstable regions and dark-gray unacceptable response regions penetrate the manufacturing tolerance circle of Fig. 12. Together, there is a $14.1 \%$ probability that the system behavior is unacceptable.

For simplicity, let us consider how zero-average mistuning, $\Sigma z_{i}=0$, affects stability and forced response. We want to understand dependence on the magnitude of mistuning. Consider a mistuning direction $\hat{z}$ with $\Sigma \hat{z}_{i}=0$ on the unit sphere $\| \hat{\mathbf{z}} \mid=1$, and let mistuning $z$ vary along this direction as $z=$ $\varepsilon \hat{z}$. From Eq. (52), each eigenvalue $\lambda_{j}^{d}(\varepsilon \hat{z})$ varies as

$$
\operatorname{Re}\left[\lambda_{j}^{d}(\varepsilon \hat{z})\right] \approx-\eta-k_{1} \varepsilon^{2}
$$

with $\eta=-\operatorname{Re}\left[\lambda_{j}^{d}(0)\right]$, the tuned damping. If $\hat{z}$ is stabilizing, then $k_{1}=k_{1}(\hat{z})>0$, else $k_{1}<0$. Destabilizing directions $\hat{z}$ yield the black regions in Fig. 12, where $\varepsilon$ becomes sufficiently large to move $\lambda_{j}^{d}(z)$ into the right-half-plane.

At resonance frequency $\omega=0.0376$, the mistuned forced response is dominated by the least-damped mode $\lambda_{\bullet}^{3}(z)$ (see Fig. 7). Hence, Eq. (66) implies

$$
\|X(\varepsilon \hat{z})\| \approx k_{0} \varepsilon /\left(\eta+k_{1} \varepsilon^{2}\right)
$$

For $\hat{z}$ stabilizing $\left(k_{1}>0\right)$, tradeoff $(72)$ is shown in Fig. 13. This curve has been noticed numerically in Ref. 19. Other researchers have determined this tradeoff by Monte Carlo simulations, but have also been unable to explain its shape. ${ }^{20}$ In our case, symmetry provides a straightforward explanation. Rotation invariance (19) implies that eigenvalues vary quadratically with mistuning [the $\eta+k_{1} \varepsilon^{2}$ term in the denominator of Eq. (72)], whereas eigenvectors are permitted to vary linearly (the $k_{0} \varepsilon$ term in the numerator). In the $\hat{z}$ stabilizing case, the response first climbs linearly as $k_{0} / \eta$ (mistuned modes appear as in Fig. 6), and then sinks quadratically as eigenvalues move into the left-half-plane. Light damping (small $\eta$ ) causes a fast linear growth.

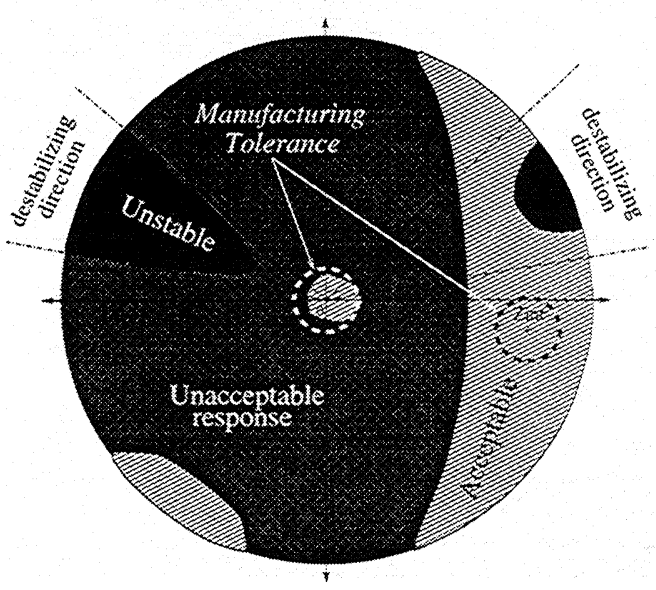

Fig. 12 Intentional mistuning for robustness under manufacturing uncertainty. Mistuning space: $z$. 


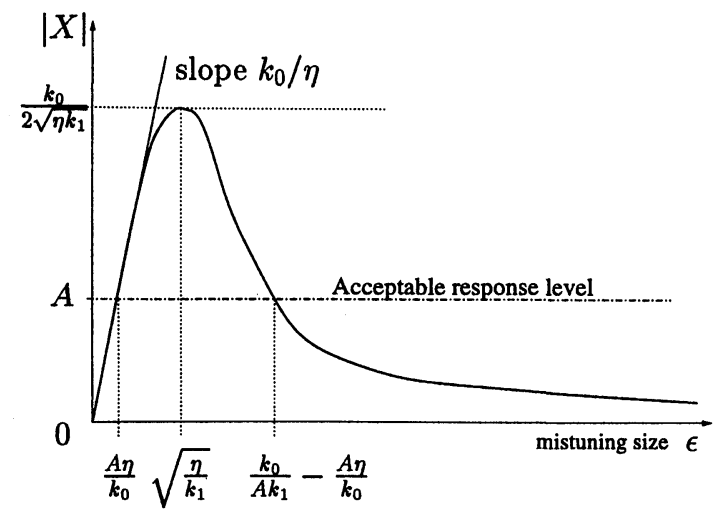

Fig. 13 Response sensitivity to mistuning size [Eq. (72)].

If we choose $A$ as the acceptable blade vibration, then the leftmost estimate of Fig. 13 yields the allowable manufacturing tolerance:

$$
\left\|z_{\text {manuf }}\right\| \leq\left(A \eta / k_{0}\right)
$$

We could find the minimum estimate [using $\max k_{0}(\hat{z})$ ] by varying $\hat{z}$ over the unit sphere $\|\hat{z}\|=1$. Equation (73) is equivalent to the tradeoff of Eq. (70).

Varying $\hat{z}$ and $\varepsilon$ together recovers Fig. 12. Coefficients $k_{0}$ and $k_{1}$ vary with $\hat{z}$, so that different directions have different sections of acceptable and unacceptable response. To pick an intentional mistuning $z_{\text {int }}$, we must find a stabilizing direction $\hat{z}$ (see Ref. 9) and pick $\varepsilon$ beyond the right-hand intersection,

$$
\varepsilon^{*}=\left(k_{0} / A k_{1}\right)-\left(A \eta / k_{0}\right)
$$

of Fig. 12: $z_{\text {int }}=\varepsilon \hat{z}$ with $\varepsilon>\varepsilon^{*}$. Now an additional small random mistuning $z=z_{\text {int }}+z_{\text {manuf }}$ will shift us along the gentle right-hand portion of Eq. (72).

Numerical results for the model of Sec. VI are shown graphically in Fig. 14. Recall that for a $2 \%$ manufacturing tolerance there is a $4.6 \%$ probability of unacceptable response. We choose a specific manufacturing mistuning $\left\|z_{\text {spec }}\right\| \leq 2 \%$, such that $X\left(z_{\text {spec }}\right)$ is unacceptable. Resulting amplitude of all the blades $\left|\boldsymbol{X}_{i}\left(z_{\text {spec }}\right)\right|$ is plotted as thin lines in Fig. 14. The mistuned response is far above the acceptable limit $A$ (dashed line):

$$
\left\|X\left(z_{\text {spec }}\right)\right\|_{\infty}=\max _{i}\left|X_{i}\left(z_{\text {spec }}\right)\right| \gg 10\|X(0)\|_{\infty}=A
$$

Now pick the intentional mistuning:

$$
z_{\text {int }}=\varepsilon \hat{z}=0.1(1,1,1,1,1,0,-1,-1,-1,-1,-1)
$$
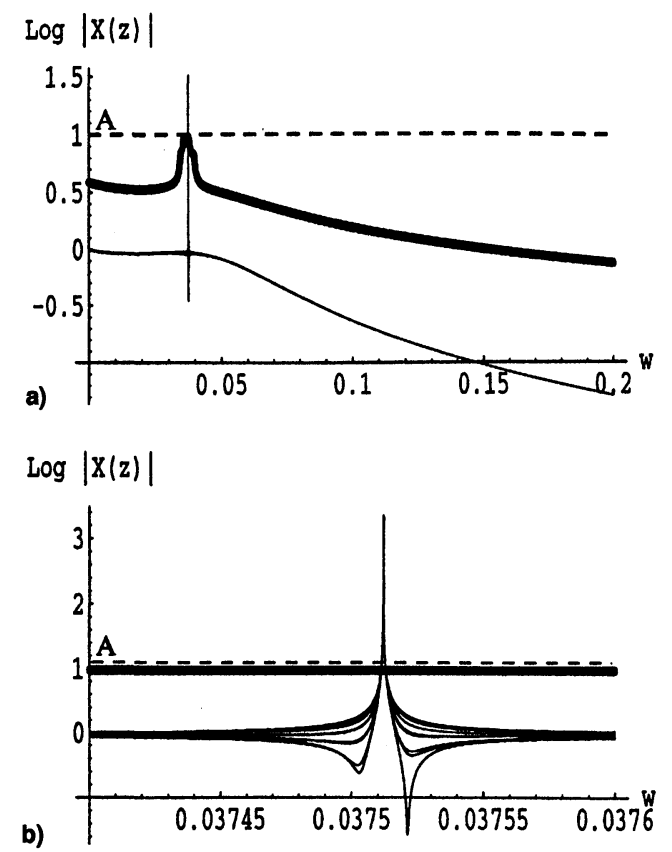

Fig. 14 a) Response under manufacturing tolerance and b) with zoom. Acceptable level $A=---$, bound for $\left|X\left(z_{\text {int }}+z_{\text {manuf }}\right)\right|=$ , amplitude $\left|X\left(z_{\text {spec }}\right)\right|$ of all blades $=$

\section{Conclusions}

This paper presents a perturbation scheme to find the forced response as a function of mistuning. By using symmetry arguments and an eigenstructure perturbation we are able to accurately predict forced response as a function of mistuning for any model. Our method reveals the underlying tradeoffs between forcing frequency, damping, resonance, and robustness. It is shown that intentional mistuning can lead to improved performance (both stability and forced response) under manufacturing uncertainty. As a result, intentional mistuning may be a viable technique for increasing safety and performance in jet engines.

\section{Appendix: Technical Results}

Here we prove some of the more technical results in the paper. First we show a symmetry result for trajectories.

Theorem A.1: For model (1) with symmetry (5), the trajectories $\boldsymbol{\xi}(\hat{\boldsymbol{x}}, s, z, t)$ satisfy condition (6).

Proof: Let $\boldsymbol{\eta}(t)=\varphi \boldsymbol{\xi}(\hat{\boldsymbol{x}}, s, \boldsymbol{z}, t)-\boldsymbol{\xi}(\varphi \hat{\boldsymbol{x}}, s+\tau, \varphi \boldsymbol{z}, t+$ $\tau)$. By construction, $\eta(s)=\varphi \hat{\boldsymbol{x}}-\varphi \hat{\boldsymbol{x}}=0$. From Eq. (1) we have

$$
\begin{aligned}
\dot{\boldsymbol{\eta}}(t) & =\varphi \boldsymbol{\xi}(\hat{\boldsymbol{x}}, s, z, t)-\boldsymbol{\xi}(\varphi \hat{\boldsymbol{x}}, s+\tau, \varphi z, t+\tau) \quad \text { by construction } \\
& =\varphi \boldsymbol{f}[\boldsymbol{\xi}(\hat{\boldsymbol{x}}, s, z, t), z, t]-\boldsymbol{f}[\boldsymbol{\xi}(\varphi \hat{\boldsymbol{x}}, s+\tau, \varphi z, t+\tau), \varphi z, t+\tau] \quad \text { from Eq. (1) } \\
& =\boldsymbol{f}[\varphi \xi(\hat{\boldsymbol{x}}, s, z, t), \varphi z, t+\tau]-\boldsymbol{f}[\boldsymbol{\xi}(\varphi \hat{\boldsymbol{x}}, s+\tau, \varphi z, t+\tau), \varphi z, t+\tau] \quad \text { symmetry (5) } \\
& =\boldsymbol{f}[\boldsymbol{\eta}(t)+\boldsymbol{\xi}(\varphi \hat{\boldsymbol{x}}, s+\tau, \varphi z, t+\tau), \varphi z, t+\tau]-f[\xi(\varphi \hat{\boldsymbol{x}}, s+\tau, \varphi z, t+\tau), \varphi z, t+\tau] \quad \text { by construction } \\
& \triangleq \boldsymbol{H}(\eta, t)
\end{aligned}
$$

Here, $\hat{z}$ is chosen by an eigenvalue optimization as in Ref. 9, whereas $\varepsilon=10 \%$ is larger than $\varepsilon^{*}$ of Eq. (74). Using the triangle inequality, Eqs. (43), (52), (66), and (68) provide bounds for stability $\operatorname{Re}\left[\lambda\left(z_{\text {int }}+z_{\text {manuf }}\right)\right]$ and forced response $\left\|X\left(z_{\text {int }}+z_{\text {manuf }}\right)\right\|_{\infty}$ for all manufacturing mistuning $\left\|z_{\text {manuf }}\right\| \leq$ $2 \%$. The forced response bound is shown as the thick line in Fig. 14. Clearly, it falls below $A$ for all forcing frequencies $\omega$. Thus, the introduction of intentional mistuning $z_{\text {int }}$ guarantees stability and acceptable response under manufacturing tolerance.
Clearly, $\boldsymbol{H}(0, t)=0$ for all $t$, regardless of values $\hat{\boldsymbol{x}}, s$, and $\boldsymbol{z}$. Thus, $\boldsymbol{\eta}(t)$ satisfies $\dot{\boldsymbol{\eta}}=\boldsymbol{H}(\boldsymbol{\eta}, t), \boldsymbol{\eta}(s)=0$ with $\boldsymbol{H}(0, t) \equiv 0$, and so $\boldsymbol{\eta}$ starts at zero and stays zero for all time. Consequently, $\boldsymbol{\eta}(t)=\varphi \boldsymbol{\xi}(\hat{\boldsymbol{x}}, s, z, t)-\boldsymbol{\xi}(\varphi \hat{\boldsymbol{x}}, s+\tau, \varphi z, t+\tau)=0$ for all $\hat{\boldsymbol{x}}, s, z$, and $\boldsymbol{t}$.

Now we show that asymmetric equilibria come in families of size $r$ (result also found in Ref. 14).

Lemma A.1: Given the tuned unforced system $\hat{\boldsymbol{f}}(\boldsymbol{x}, 0)$ with the symmetry $\varphi \bar{f}(x, 0)=\bar{f}(\varphi x, 0)$, consider an asymmetric equilibrium point $\boldsymbol{x}^{*}(0) \neq \varphi \boldsymbol{x}^{*}(0)$. Then $\varphi^{j} \boldsymbol{x}^{*}(0)$ for $j \in(1,2$, $\ldots, r)$ are also equilibria of $\bar{f}(\boldsymbol{x}, 0)$. 
of $\bar{f}$

$$
\bar{f}\left[\varphi^{j} \boldsymbol{x}^{*}(0), 0\right]=\varphi^{j} \bar{f}\left[\boldsymbol{x}^{*}(0), 0\right]=0
$$

Hence, $\varphi^{j} \boldsymbol{x}^{*}(0)$ form a total of $r$ equilibrium points.

Next we show an equilibrium symmetry.

Lemma A.2: Consider the unforced system $\dot{\boldsymbol{x}}=\overline{\boldsymbol{f}}(\boldsymbol{x}, \boldsymbol{z})$ [see Eq. (9)], with symmetry $\varphi \overline{\boldsymbol{f}}(\boldsymbol{x}, \boldsymbol{z})=\overline{\boldsymbol{f}}(\varphi \boldsymbol{x}, \varphi z)$ and equilibrium point $x^{*}(z)$. If the tuned equilibrium point is symmetric $x^{*}(0)$ $=\varphi x^{*}(0)$ and the Jacobian at $x^{*}(0)$ is nonsingular,

$$
\operatorname{det}\left\{\frac{\partial \bar{f}}{\partial x}\left[x^{*}(0), 0\right]\right\} \neq 0
$$

(true almost always), then the equilibrium has symmetry $\varphi x^{*}(z)=x^{*}(\varphi z)$

Proof: Let $\boldsymbol{\eta}(z)=x^{*}(z)-x^{*}(0)$. Because $x^{*}(z)$ is an equilibrium point that varies as $z$ changes, it satisfies $\bar{f}\left[x^{*}(z), z\right]=$ 0 for any $z$, specifically:

$$
\bar{f}\left[x^{*}(z), z\right]=\bar{f}[x *(\varphi z), \varphi z]=0
$$

By symmetry $\varphi \bar{f}(x, z)=\bar{f}(\varphi x, \varphi z)$ of the unforced system, we have

$$
\bar{f}\left[x^{*}(z), z\right]=\varphi \bar{f}\left[\varphi^{-1} x^{*}(\varphi z), z\right]=0
$$

Substituting $\boldsymbol{\eta}$ and noting assumption $\varphi^{-1} \boldsymbol{x}^{*}(0)=\boldsymbol{x}^{*}(0)$ yields

$$
\bar{f}\left[x^{*}(0)+\boldsymbol{\eta}(z), z\right]=\varphi \bar{f}\left[x^{*}(0)+\varphi^{-1} \boldsymbol{\eta}(\varphi z), z\right]=0
$$

Because $\operatorname{det}\{(\partial \bar{f} / \partial x)[x *(0), 0]\} \neq 0$, the implicit function theorem (Ref. 21, p. 354) implies that relation $\bar{f}\left[x^{*}(0)+\boldsymbol{\alpha}(z), z\right]$ $=0$ uniquely defines $\boldsymbol{\alpha}(z)$. (Note that $\varphi \overline{\boldsymbol{f}}=0$ implies $\overline{\boldsymbol{f}}=0$.) Hence, $\boldsymbol{\eta}(z)=\varphi^{-1} \eta(\varphi z)$, and so

$$
\varphi x^{*}(z)=x^{*}(0)+\varphi \boldsymbol{\eta}(z)=x^{*}(0)+\boldsymbol{\eta}(\varphi z)=x^{*}(\varphi z)
$$

The following results state linear symmetries that follow from nonlinear symmetry [Eq. (5)] and the equilibrium symmetry of Lemma A.2. First we prove Theorem 3.1.

Proof: To prove the theorem, we need to show $\bar{M} V_{j}^{d}=$ $\lambda_{j}^{d} \boldsymbol{V}_{j}^{d}$ and $\boldsymbol{U}_{j}^{d} \bar{M}=\lambda_{j}^{d} \boldsymbol{U}_{j}^{d}$. Both statements are verified trivially by substitution.

Lemma A. 3 proves symmetry of the linearization $M(z)$ found in Eq. (13).

Lemma A.3: Consider unforced system $\bar{f}$ with symmetry $\bar{f}(x, z)=\varphi^{-1} \bar{f}(\varphi x, \varphi z)$, and equilibrium point $x^{*}$ with symmetry $x^{*}(z)=\varphi^{-1} x^{*}(\varphi z)$ of Table 1 . Then $M(z)$ defined in Eq. (12) has symmetry $M(z)=\varphi^{-1} M(\varphi z) \varphi$.

Proof: By definition of the equilibrium point $x^{*}, \bar{f}\left[x^{*}(z), z\right]$ $=0$. Hence, $\mathrm{a}$ Taylor expansion yields

$$
\bar{f}(x, z)=[M(z)]\left[x-x^{*}(z)\right]+\mathscr{O}\left[\left\|x-x^{*}(z)\right\|^{2}\right]
$$

Applying symmetry of $\bar{f}$ to the linear term provides

$$
\begin{aligned}
\varphi \bar{f}(x, z) & =\bar{f}(\varphi x, \varphi z) \\
\Rightarrow \varphi\left\{[M(z)]\left[x-x^{*}(z)\right]\right\} & =[M(\varphi z)]\left[\varphi x-x^{*}(\varphi z)\right]
\end{aligned}
$$

The crucial step is the equilibrium symmetry $\varphi x^{*}(z)=x^{*}(\varphi z)$, which allows

$$
\varphi\left\{[M(z)]\left[x-x^{*}(z)\right]\right\}=[M(\varphi z)] \varphi\left[x-x^{*}(z)\right]
$$

So, for any $\boldsymbol{y}=\mathbf{x}-x^{*}(z)$, we have $M(z) y=\varphi^{-1} M(\varphi z) \varphi y$, the required symmetry.
Next we show symmetry of the forcing vectors $\boldsymbol{B}_{l}$.

Lemma A.4: Forcing term $\boldsymbol{f}^{\prime}$ has symmetry (5), namely,

$$
\boldsymbol{f}^{\prime}(\boldsymbol{x}, z, t)=\varphi^{-1} \boldsymbol{f}^{\prime}(\varphi x, \varphi z, t+\tau)
$$

Hence, symmetry $\varphi \boldsymbol{x}^{*}(\boldsymbol{z})=\boldsymbol{x} *(\varphi z)$ on the equilibrium implies that any vector coefficient $\boldsymbol{B}_{l}(z)$ has symmetry $\boldsymbol{B}_{l}(z)=p_{l}$ $\varphi^{-1} \boldsymbol{B}_{l}(\varphi z)$.

Proof: By definition of $\boldsymbol{B}_{l}$, setting all disturbance modes to zero except mode $l$ reduces $f^{\prime}$ to

$$
f^{\prime}[\boldsymbol{x} *(z), z, t]=\boldsymbol{B}_{l}(z) e^{i l \Omega t}
$$

By symmetry on $f^{\prime}$ and $x^{*}$, we have

$$
\begin{aligned}
\varphi f^{\prime}\left[x^{*}(z), z, t\right] & =f^{\prime}\left[\varphi x^{*}(z), \varphi z, t+\tau\right] \\
& =f^{\prime}\left[x^{*}(\varphi z), \varphi z, t+\tau\right]
\end{aligned}
$$

Substituting Eq. (A1) into (A2) yields

$$
\begin{aligned}
\varphi \boldsymbol{B}_{l}(z) e^{i l \Omega t} & =\boldsymbol{B}_{l}(\varphi z) e^{i l \Omega(t+\tau)} \\
& =p_{l} \boldsymbol{B}_{l}(\varphi z) e^{i l \Omega t}
\end{aligned}
$$

because $e^{i l \Omega \tau}=e^{2 \pi i l / r} \triangleq p_{l}$. Hence, $\boldsymbol{B}_{l}(z)=p_{l} \varphi^{-1} \boldsymbol{B}_{l}(\varphi z)$.

Symmetry of $M(z)$ in Lemma A.3 implies a symmetry on eigenvalues and eigenvectors.

Lemma A.5: If the Jacobian has symmetry $M(z)=$ $\varphi^{-1} M(\varphi z) \varphi$, then its eigenvalues $\lambda_{i}(z)$ have symmetry $\lambda_{i}(z)=$ $\lambda_{i}(\varphi z)$.

Proof: An eigenvalue $\lambda_{i}(z)$ satisfies $M(z) \boldsymbol{V}(z)=\lambda_{i}(z) \boldsymbol{V}(z)$, where $\boldsymbol{V}(z)$ is the corresponding right eigenvector. By symmetry

$$
\begin{array}{rlrl}
M(z) \boldsymbol{V}(z) & =\lambda_{i}(z) \boldsymbol{V}(z) \\
\varphi[M(z) \boldsymbol{V}(z)] & =\lambda_{i}(z) \varphi V(z) & & \text { rotate both sides } \\
M(\varphi z)[\varphi V(z)] & =\lambda_{i}(z) \varphi V(z) & & \text { by symmetry of } M
\end{array}
$$

So, $\lambda_{i}(z)$ is also an eigenvalue of $M(\varphi z)$ with eigenvector $\varphi \boldsymbol{V}(z)$; hence, by definition, there exists an eigenvalue $\lambda_{j}(\varphi z)$ of $M(\varphi z)$ with $\lambda_{i}(z)=\lambda_{j}(\varphi z)$. Setting $z=0$ we see that these must be the same eigenvalue $i=j$. Consequently, $\lambda_{i}(z)=$ $\lambda_{i}(\varphi z)$.

A similar result holds for right and left eigenvectors.

Lemma A.6: For Jacobian with symmetry $M(z)=$ $\varphi^{-1} M(\varphi z) \varphi$, right and left eigenvectors, $\boldsymbol{V}_{j}^{d}$ and $\boldsymbol{U}_{j}^{d}$, in the notation of Theorem 3.1 have symmetry:

$$
\begin{gathered}
\boldsymbol{V}_{j}^{d}(z)=p_{j} \varphi^{-1} \boldsymbol{V}_{j}^{d}(\varphi z) \\
\boldsymbol{U}_{j}^{d}(z)=p_{j}^{-1} \varphi^{-1} \boldsymbol{U}_{j}^{d}(\varphi z)
\end{gathered}
$$

Proof: By definition:

$$
\begin{gathered}
M(z) \boldsymbol{V}_{j}^{d}(z)=\lambda_{j}^{d}(z) V_{j}^{d}(z) \\
\varphi\left[M(z) V_{j}^{d}(z)\right]=\lambda_{j}^{d}(z) \varphi V_{j}^{d}(z) \quad \text { rotate both sides } \\
M(\varphi z)\left[\varphi V_{j}^{d}(z)\right]=\lambda_{j}^{d}(\varphi z) \varphi V_{j}^{d}(z) \quad \text { by symmetry of } M \text { and } \lambda
\end{gathered}
$$

So, $\varphi V_{j}^{d}(z)$ is the $j, d$ th right eigenvector of $M(\varphi z)$. Because eigenvectors are unique up to a scaling constant, we get $\varphi \boldsymbol{V}_{j}^{d}(\boldsymbol{z})=\alpha \boldsymbol{V}_{j}^{d}(\varphi z)$. Setting $z=0$, we find $\alpha=p_{j}$, because $\varphi \boldsymbol{V}_{j}^{d}(0)=p_{j} \boldsymbol{V}_{j}^{d}(0)$ from Theorem 3.1. A mirror arguments holds for left eigenvectors $\boldsymbol{U}_{j}^{d}(z)$.

To conclude this portion we show the symmetry of the forced response $\boldsymbol{X}(\mathbf{z})$. This follows from symmetry (5), by way of Theorem A.1, and the equilibrium symmetry of Lemma A.2.

Lemma A.7: If model $f$ has symmetry (5) and equilibrium point $\boldsymbol{x}^{*}$ has the symmetry of Lemma A.2, then the linear forced response $\boldsymbol{X}$ of (13) has symmetry $\boldsymbol{X}(z)=p_{1} \varphi^{-1} \boldsymbol{X}(\varphi z)$.

Proof: By Theorem A.1, trajectories $\xi$ have symmetry:

$$
\boldsymbol{\xi}(\hat{\boldsymbol{x}}, s, z, t)=\varphi^{-1} \boldsymbol{\xi}(\varphi \hat{\boldsymbol{x}}, s+\tau, \varphi z, t+\tau)
$$


For stable $M(z)$, as assumed in Sec. III.B, the steady-state response is given by

$$
\xi(z, t)=x^{*}(z)+X(z) e^{i l \Omega t}
$$

where initial conditions $\hat{\boldsymbol{x}}, s$ may be dropped because the transient decays to zero. By symmetry of $\boldsymbol{\xi}$ we have

$$
\begin{aligned}
& \varphi \boldsymbol{\xi}(z, t)=\varphi x^{*}(z)+\varphi X(z) e^{i l \Omega t}=\xi(\varphi z, t+\tau)=x^{*}(\varphi z) \\
& +X(\varphi z) e^{i l \Omega(t+\tau)}
\end{aligned}
$$

However, $\varphi x^{*}(z)=x^{*}(\varphi z)$, and so,

$$
\varphi X(z)=X(\varphi z) e^{i l \Omega \tau} \triangleq p_{l} X(\varphi z)
$$

Finally, we show that the forced response $X(z)$ of Eq. (13) is smooth in $z$ (no bifurcation) at $\hat{z}$ if $M(\hat{z})$ has no eigenvalues on the imaginary axis. Because $M(\hat{z})$ has no purely imaginary eigenvalues, $A(\hat{z})=[i \omega I-M(\hat{z})]$ of Eq. (33) has no zero eigenvalues and is invertible $\left[A^{-1}(\hat{z})\right.$ exists]. From Eq. (13) we note that

$$
\boldsymbol{X}(\hat{z})=A^{-1}(\hat{z}) \boldsymbol{B}_{l}(\hat{z})
$$

Our analysis assumes that $M(z)$ and $\boldsymbol{B}_{l}(z)$ are smooth. Hence, to show that $\boldsymbol{X}(\hat{z})$ is smooth in Eq. (A4), we need only show $A^{-1}(z)$ is smooth at $\hat{z}$. This follows from Theorem A.2.

Theorem A.2: Consider a matrix $A(\alpha)$ dependent on real scalar $\alpha$ with $A(\alpha)$ smooth and invertible for all $\alpha$ in $\left(\alpha_{1}, \alpha_{2}\right)$, so that $\mathrm{d} A(\alpha) / \mathrm{d} \alpha$ and $A^{-1}(\alpha)$ exist for all $\alpha \in\left(\alpha_{1}, \alpha_{2}\right)$. Then, $\mathrm{d} A^{-1}(\alpha) / \mathrm{d} \alpha$ exists for all $\alpha \in\left(\alpha_{1}, \alpha_{2}\right)$, meaning $A^{-1}(\alpha)$ is smooth for all $\alpha$ values in the open interval $\left(\alpha_{1}, \alpha_{2}\right)$.

Proof: The proof is standard and proceeds by construction. For any $\alpha \in\left(\alpha_{1}, \alpha_{2}\right)$, we have

$$
\frac{\mathrm{d}}{\mathrm{d} \alpha}\left[A^{-1}(\alpha) A(\alpha)\right]=\frac{\mathrm{d} I}{\mathrm{~d} \alpha}=0
$$

By the product rule

$$
\left[\frac{\mathrm{d} A^{-1}}{\mathrm{~d} \alpha}(\alpha)\right][A(\alpha)]+\left[A^{-1}(\alpha)\right]\left[\frac{\mathrm{d} A}{\mathrm{~d} \alpha}(\alpha)\right]=0
$$

and so

$$
\frac{\mathrm{d} A^{-1}}{\mathrm{~d} \alpha}(\alpha)=-\left[A^{-1}(\alpha)\right]\left[\frac{\mathrm{d} A}{\mathrm{~d} \alpha}(\alpha)\right]\left[A^{-1}(\alpha)\right]
$$

which exists for all $\alpha \in\left(\alpha_{1}, \alpha_{2}\right)$ because $A^{-1}(\alpha)$ and $\mathrm{d} A(\alpha) /$ $\mathrm{d} \alpha$ exist for all $\alpha$ in $\left(\alpha_{1}, \alpha_{2}\right)$.

Theorem A. 2 extends to vector dependence, $\alpha \mapsto z$, by the chain rule. Hence, $M(\hat{z})$, having no eigenvalues on the imaginary axis, implies that $A^{-1}(z)$ is smooth at $\hat{z}$. So, $\boldsymbol{X}(\boldsymbol{z})$ is smooth at $\hat{z}$ by Eq. (A4). No bifurcations occur unless $M(\hat{z})$ has eigenvalues on the imaginary axis.

\section{Acknowledgments}

This research was supported, in part, by an NSF Fellowship and AFOSR Grant F49620-95-1-0409. I wish to thank Richard Murray at Caltech for his help and interest. Thanks also go to
Daniel Gysling and Gonzalo Rey at United Technologies Research Center for their support during the summer of 1997.

\section{References}

1"Report of the Ad Hoc Committee on Air Force Aircraft Jet Engine Manufacturing and Production Processes," U.S. Air Force Scientific Advisory Board, Tech, Rept., July 1992.

${ }^{2}$ Davenport, O., “AFOSR High Cycle Fatigue Workshop," Meeting Briefing, U.S. Air Force Office of Scientific Research, Oct. 1995.

${ }^{3}$ Dye, R. C. F., and Henry, T. A., "Vibration Amplitudes of Compressor Blades Resulting from Scatter in Blade Natural Frequencies," Journal of Engineering for Power, Vol. 91, July 1969, pp. 182-188.

${ }^{4}$ Mehmed, O., and Murthy, D. V., "Experimental Investigation of Propfan Aeroelastic Response in Off-Axis Flow with Mistuning," AIAA Paper 88-3153, July 1988.

${ }^{5}$ Bendiksen, O. O., "Flutter of Mistuned Turbomachinery Rotors," Journal of Engineering for Gas Turbines and Power, Vol. 106, 1984, pp. 25-33.

${ }^{6}$ Crawley, E. F., and Hall, K. C., "Optimization and Mechanisms of Mistuning in Cascades," American Society of Mechanical Engineers, Paper 84-GT-196, 1984.

${ }^{7}$ Kaza, K. R., and Kielb, R. E., "Flutter and Response of a Mistuned Cascade in Incompressible Flow," AIAA Journal, Vol. 20, No. 8, 1982 , pp. $1120-1127$.

${ }^{8}$ Whitehead, D. S., "Torsional Flutter of Unstalled Cascade Blades at Zero Deflection," Aeronautical Research Council, R\&M 3429 , 1964.

9'Shapiro, B., "Symmetry Approach to Extension of Flutter Boundaries via Mistuning," Journal of Propulsion and Power, Vol. 14, No. 3, 1998, pp. 354-366.

${ }^{10}$ Pierre, C., and Murthy, D. V., "Aeroelastic Modal Characteristics of Mistuned Blade Assemblies: Mode Localization and Loss of Eigenstructure," AIAA Journal, Vol. 30, No. 10, 1992, pp. 2483-2496.

"Watson, B. C., "An Investigation into the Influence of Mistuning on the Forced Response of Bladed Disk Assemblies," Ph.D. Dissertation, Georgia Inst. of Technology, Atlanta, GA, 1993.

${ }^{12}$ Wei, S. T., and Pierre, C., "Statistical Analysis of the Forced Response of Mistuned Cyclic Assemblies," AIAA Journal, Vol. 28 , No. 5, 1990, pp. 861-868.

${ }^{13}$ Sinha, A., "Calculating the Statistics of Forced Response of a Mistuned Bladed Disk Assembly," AIAA Journal, Vol. 24, 1986, pp. $1797-1801$.

${ }^{14}$ Golubitsky, M., Stewart, I., and Schaeffer, D. G., Singularities and Groups in Bifurcation Theory, Vol. 2, Springer-Verlag, New York, 1988

${ }^{15}$ Mignolet, M. P., and Hu, W., "Direct Prediction of the Effects of Mistuning on the Forced Response of Bladed Disks," Proceedings of Gas Turbine and Aeroengine Congress and Exhibition (Orlando, FL), American Society of Mechanical Engineers, New York, 1997.

${ }^{16}$ Lancaster, P., "On Eigenvalues of Matrices Dependent on a Parameter," Numerische Mathematik, Vol. 6, 1964, pp. 377-387.

${ }^{17}$ Afolabi, F., and Nwokah, O. D. I., "The Frequency Response of Mistuned Cyclic Systems," Proceedings of Vibration Analysis - Techniques and Applications; 12th Biennial American Society of Mechanical Engineers Conference on Mechanical Vibration and Noise (Montreal, Canada), American Society of Mechanical Engineers, New York, 1989, pp. 209-217.

${ }^{18}$ Shapiro, B., and Coller, B. D., "Characterizing Optimal Mistuning by Symmetry Arguments,"' IEEE Conference on Control Applications, Inst. of Electrical and Electronics Engineers, New York, 1997.

${ }^{19}$ Kruse, M. J., and Pierre, C., "Dynamic Response of an Industrial Turbomachinery Rotor," Proceedings of the AIAA/ASME/SAE/ASEE 32nd Joint Propulsion Conference and Exhibit (Lake Buena Vista, FL), AIAA, Reston, VA, 1996

${ }^{20}$ Castanier, M. P., and Pierre, C., "Consideration on the Benefits of Intentional Blade Mistuning for the Forced Response of Turbomachinery Rotors," Proceedings of the ASME Aerospace Division, Vol. AD-55, American Society of Mechanical Engineers, New York, 1997, pp. 419-425.

${ }^{21}$ Protter, M. H., and Morrey, C. B., A First Course in Real Analysis, Springer-Verlag, New York, 1977. 